\title{
XMM-Newton study of the two-dimensional structure of the REFLEX-DXL galaxy clusters ${ }^{\star}$
}

\author{
A. Finoguenov, H. Böhringer, and Y.-Y. Zhang
}

\author{
Max-Planck-Institut für extraterrestrische Physik, Giessenbachstraße, 85748 Garching, Germany \\ e-mail: alexis@xray.mpe.mpg.de
}

Received 25 April 2005 / Accepted 20 June 2005

\section{ABSTRACT}

We carry out a two-dimensional study of temperature, entropy, and pressure distributions in a nearly volume-limited subsample of REFLEX clusters at redshift 0.3 , the REFLEX-DXL. We use the observations gained by XMM-Newton, which cover the central $1-2 \times r_{500}$. We define the substructure in both entropy and pressure as a deviation from the mean profile of the sample. The non-parametric locally weighted regression suggests a broken power-law approximation to the entropy profile with inner and outer slopes of 0.78 and 0.52 , respectively, and a break at $0.5 r_{500}$. Characterization of the pressure profile is more complex, requiring three power laws, with slopes -0.64 at $r<0.3 r_{500},-2.47$ at $r>0.5 r_{500}$ and a slope of -1.50 in between. Analysis of the substructure in the pressure and entropy maps reveals somewhat larger fluctuations around the mean pressure profile compared to the entropy. Typically, pressure fluctuations are found at the $30 \%$ level, while the entropy fluctuations are at the $20 \%$ level (rms). We compare the cumulative distribution of the substructure level in the REFLEX-DXL sample with the results of numerical simulation, and by means of KS test, show that they are in agreement. A discussion of the origin of the substructure is provided on an individual cluster basis.

Key words. cosmology: observations - galaxies: clusters: general

\section{Introduction}

Use of clusters of galaxies for cosmological tests in present and future surveys rely on an understanding of cluster physics. A number of observational and theoretical efforts have been devoted to studying the effects of non-gravitational heating in explaining the observed scaling of clusters with mass (see Voit 2005, for a review). Another long recognized issue consists of the effect of merging on the appearance of clusters (e.g. Randall et al. 2002; Rowley et al. 2004). It is widely accepted that the latter effect dominates the appearance of the most massive clusters, where it also could be more pronounced, due to the dependence of the accretion history of clusters on their mass. Thus, a study of the dynamical state of a representative sample of massive clusters appears to be well-grounded. In this paper we explore the structure of individual clusters from a volumelimited sample of most massive clusters at a redshift of 0.3 to provide an in-depth coverage of the properties that define both the X-ray luminosity and the thermal Sunyaev-Zel'dovich effect (SZE) of clusters.

\subsection{The REFLEX-DXL sample}

The REFLEX-DXL galaxy cluster sample, comprising distant X-ray luminous objects within REFLEX, was constructed

* Figures 6 to 13 are only available in electronic form at http//www. edpsciences.org from the REFLEX galaxy cluster survey covering the ROSAT detected galaxy clusters above a flux limit of $3 \times$ $10^{-12} \mathrm{erg} \mathrm{s}^{-1} \mathrm{~cm}^{-2}$ in the 0.1 to $2.4 \mathrm{keV}$ band in an $4.24 \mathrm{ster}$ region of the southern sky (see Böhringer et al. 2001a for details). The REFLEX-DXL clusters form a nearly volumelimited subset of REFLEX in the redshift range 0.27 to 0.31 including 13 members plus a fourteenth cluster which was previously assigned to the sample but had to be excluded after a revision of the optically determined redshift. We included this cluster here since it fits nicely into the homogeneous observational data properties of the REFLEX-DXL sample. All clusters exceed a luminosity of $10^{45} h_{50}^{-1} \mathrm{erg} \mathrm{s}^{-1}$ in the ROSAT band ( 0.1 to $2.4 \mathrm{keV})$. For five of the clusters, the XMM observations were affected by strong proton flares. Their re-observation, which will be reported elsewhere, provided us with good results except for one cluster, RXCJ2011-5725. One of the clusters included in the present study, RXCJ0658-5557, was a verification phase target with a significantly longer nominal observing time. Further properties of the REFLEX-DXL clusters are described in Zhang et al. (2004) and Böhringer et al. (2005, in prep.).

In Table 1 we summarize the properties of the clusters used in this study. Column 1 gives the RXCJ designation of the source, 2-4 optical redshift, corresponding luminosity distance, and the conversion factor from apparent angular to physical scale (calculated for $\Omega_{\mathrm{M}}=1-\Omega_{\Lambda}=0.3$, and a Hubble constant of $70 \mathrm{~km} \mathrm{~s}^{-1} \mathrm{Mpc}^{-1}$ ). Column 5 gives the measured 
Table 1. Scaling assumptions for the analyzed clusters.

\begin{tabular}{cccccccc}
\hline \hline $\begin{array}{c}\text { RXCJ } \\
\text { name }\end{array}$ & $z$ & $\begin{array}{c}D_{l} \\
\mathrm{Mpc}\end{array}$ & $\begin{array}{c}\text { plate } \\
\text { scale } \\
\mathrm{kpc} /\end{array}$ & $\begin{array}{c}\text { cluster center } \\
\text { RA, Dec } \\
\text { FK5 (Eq.J2000) }\end{array}$ & $\begin{array}{c}k T_{\mathrm{ew}} \\
\mathrm{keV}\end{array}$ & $\begin{array}{c}r_{500} \\
\mathrm{Mpc}\end{array}$ & $\begin{array}{c}\text { other } \\
\text { names }\end{array}$ \\
\hline $0014.3-3022$ & 0.3066 & 933 & 271 & $00: 14: 19.288-30: 23: 07.41$ & $8.3 \pm 0.4$ & 1.11 & A2744, AC118 \\
$0043.4-2037$ & 0.2924 & 903 & 263 & $00: 43: 24.446-20: 37: 30.72$ & $6.8 \pm 0.4$ & 1.01 & A2813 \\
$0232.2-4420$ & 0.2836 & 884 & 257 & $02: 32: 18.561-44: 20: 48.40$ & $7.6 \pm 0.4$ & 1.07 & \\
$0307.0-2840$ & 0.2578 & 825 & 240 & $03: 07: 02.084-28: 40: 00.21$ & $6.6 \pm 0.3$ & 1.01 & A3088 \\
$0528.9-3927$ & 0.2839 & 885 & 257 & $05: 28: 52.731-39: 28: 24.82$ & $7.7 \pm 0.6$ & 1.08 & \\
$0532.9-3701$ & 0.2747 & 864 & 251 & $05: 32: 56.043-37: 01: 33.52$ & $7.8 \pm 0.6$ & 1.09 & \\
$0658-5557$ & 0.2965 & 912 & 265 & $06: 58: 31.453-55: 56: 16.74$ & $12.3 \pm 0.3$ & 1.37 & 1 ES0657-558 \\
$1131.9-1955$ & 0.3075 & 935 & 272 & $11: 31: 55.742-19: 55: 42.82$ & $7.4 \pm 0.5$ & 1.05 & A1300 \\
$2337.6+0016$ & 0.2779 & 871 & 253 & $23: 37: 38.323+00: 16: 05.07$ & $7.5 \pm 0.4$ & 1.07 & A2631 \\
\hline
\end{tabular}

position of the cluster center, used in analysis of cluster structure; 6 is the mean temperature $T_{w}$ from Table 4 of Zhang et al. (2004), derived using the $0.5^{\prime}<r<4^{\prime}$ region and the energy band 1-10 keV. For the bullet cluster (RXCJ0658-5557), we used the 0.4-10 keV band and removed the soft excess by an additional spectral component for reasons explained in detail below. 7 is $r_{500}$ used for the scaling plots (an estimated radius encompassing the density equal to $500 \rho_{\text {crit }}$ ), while 8 lists the other names of the clusters.

Calculation of $r_{500}$ follows $r_{500}=0.45 \mathrm{Mpc} \times$ $\sqrt{k T_{w} / \mathrm{keV}} h_{70}^{-1} h(z)^{-1}$, where the scaling in Finoguenov et al. (2001) for $h_{50}\left(r_{500} \approx 0.63 \mathrm{Mpc} \sqrt{k T / \mathrm{keV}}\right)$ is translated into our assumption for $h_{70}=1$. We used $h(z)=\left(\Omega_{M}(1+z)^{3}+\Omega_{\Lambda}\right)^{1 / 2}$, suitable for our choice of the cosmological model. Finoguenov et al. (2001) demonstrate that the cosmological corrections are negligible in deriving the scaling for $r_{500}$ in their sample of local clusters. These corrections are, however important for REFLEX-DXL.

The suggested modified entropy scaling reads $S \sim$ $T_{w}^{0.65 \sim 2 / 3} h(z)^{-4 / 3}$ (Ponman et al. 2003). In analyzing clusters, we also present scaled pressure plots. As entropy, $T n^{-2 / 3}$, scales as $T_{w}^{2 / 3}$, the density scales as $T_{w}^{1 / 2}$, and the pressure $T n \sim T_{w}^{3 / 2}$. Finally, correction for the evolution of the critical density is $h(z)^{2}$. Throughout the paper we use the corrections by $T_{10}=\frac{T_{w}}{10 \mathrm{keV}}$. For the temperature profiles, however, we simply correct for the $T_{w}$ to match other studies.

\section{Analysis}

The main goal of the primary data reduction was to produce soft and hard images based on merging the data obtained by all EPIC detectors. Initial steps in data reduction includes the XMMSAS event processing and light curve screening, which is similar to the approach adopted by other groups (e.g. De Luca $\&$ Molendi 2003). The background subtraction is described in Zhang et al. (2004).

With these screened photon event files we produced MOS and pn images of the individual observations in the energy bands from 0.5 to 2.0 and 2.0 to $7.5 \mathrm{keV}$ with more details available in Briel et al. (2004).

The broad-band images can be used for making visible intensity structures, and variations of the temperature of the X-ray emitting plasma by producing hardness-ratio maps, which can be uniquely translated into plasma temperature distributions. In addition, one can produce pressure and entropy maps of the plasma by combining the surface brightness map and the hardness ratio map. Useful hardness ratio maps can only be produced from smoothed surface brightness maps. A variety of different smoothing procedures exist, like top-hat smoothing, Gaussian smoothing or adaptive smoothing (e.g. Churazov et al. 1999). We applied the wavelet decomposition method, which is described in detail by Vikhlinin et al. (1998). The advantage of using wavelets consists of background removal by spatial filtering and control over the statistical significance of the detected structures. Complications arised due to splitting the image into discrete scales, which we overcame by additional smoothing applied before producing the hardness ratio map. The use of wavelets provided us with a decent method to identify the regions susceptible to temperature variations. Another important feature is the high spatial resolution achieved in detecting the structure, as wavelets do not smear the data.

For every cluster, we show the results of the broad-band image investigation: an image in the $0.5-2 \mathrm{keV}$ energy band; the hardness of the emission, deduced from the ratio of the wavelet-reconstructed images in the $0.5-2$ and $2-7.5 \mathrm{keV}$ bands; as well as the projected pressure and entropy maps, constructed using the wavelet-smoothed surface-brightness map in the $0.5-2 \mathrm{keV}$ energy range as an indicator of the electron density squared and the hardness ratio map as temperature distribution, and using the definitions of the pressure $P \sim T \times \sqrt{I}$ and of the entropy $S \sim T / \sqrt[3]{I}$. In addition to visualizing the temperature distribution through hardness ratios, we also determined the local temperatures spectroscopically.

The spectral analysis was carried out using only the pn data. Background subtraction here was more demanding and based on the XMM observations of the Chandra Deep Field South, as described in Zhang et al. (2004). We selected the 0.4-7.9 keV band for analysis of all the clusters and added a soft $(k T \sim$ $0.2 \mathrm{keV}$ ) spectral component to remove any soft excess. For the most affected system, the bullet cluster (RXCJ0658-5557), we find good agreement between the mean temperatures using this method (12.3 \pm 0.3$)$ and the $1-10 \mathrm{keV}$ band $(11.8 \pm 0.3)$. 
In the spectral analysis, we produced two masks per cluster defining the spectral extraction region, one to confirm the temperature structure combined with the image, the other - to confirm pressure and entropy structure. We combined the regions so that counting statistics are not the limiting factor in our derivation of the cluster properties. We used the wavelet-based maps to identify regions with similar X-ray colors and intensity levels. To generate the mask file for use in the subsequent spectral analysis, we sampled the changes in the intensity and hardness ratio at the precision allowed by the statistics of the data. We then examined each of the isolated regions with approximately equal color and intensity, imposing an additional criterion that the regions should be larger than the PSF width $\left(15^{\prime \prime}\right)$ and contain more than 500 counts in the background corrected pn image. When sampling the pressure and entropy structure, the region selection should be fine to achieve a nearly equal temperature within the region, as the X-ray data is only sensitive to pressure and entropy by measuring the temperature and normalization of the spectra. As a result, we used finer region selection with a reduced threshold on a number of counts (300), resulting in a higher number of regions at the expense of larger uncertainty in the parameter determination. In the paper we tabulate mostly the confirmation of the temperature structure, with the exception of RXCJ0232.2-4420 and the bullet cluster, where the other mask was more useful.

For every cluster we provide a table containing the measured values with their $\pm 1 \sigma$ errors for one parameter of interest. As all these tables are similar, we give a single description for all here. Column 1 labels the region according to region selection shown in the accompanied figure, Col. 2 lists the temperature in $\mathrm{keV}$. Derived quantities that use an estimate of the projected length, as described below are reported in Cols. 3-6. These are electron density, entropy, pressure, and the (local) gas mass. Columns $7-8$ report the minimal $\left(r_{\min }\right)$ and maximal $\left(r_{\max }\right)$ radii of the extraction area, Col. 9 provides remarks on the region.

For this detailed analysis we also performed an estimate of the projection length of each analyzed region to obtain actual gas properties at these locations, as described at length in Henry et al. (2004) and Mahdavi et al. (2005). To avoid the importance of the projection effects, we discarded the regions with a ratio of the minimal to the maximal radii of values exceeding 0.8 .

\section{Results}

The statistics achieved in the observation of the REFLEX-DXL clusters only allow us to recognize the strongest fluctuations in either temperature, entropy, or pressure. In presenting results we indicate the features seen on the hardness-ratio-based maps and discuss how possible it is to confirm them through direct spectroscopic analysis. In selecting the regions according to their properties or according to their statistics, we implicitly perform an adaptive filtering of the signal. It is therefore important to characterize the spatial frequencies sampled in the analysis, which is also a way to characterize the analysis carried out and the cluster spatial scales sampled. In Fig. 1 we present an analysis showing that the choice of the regions could be characterized by a grid in cylindrical coordinates sampling

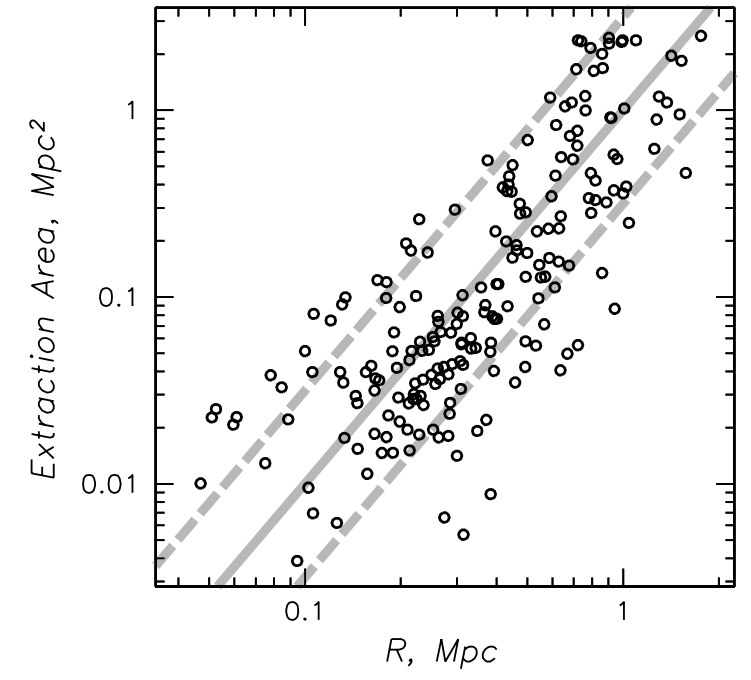

Fig. 1. Characterization of the ability of the current masking technique to reveal spatial variations in the spectral properties of gas as a function of clustercentric distance (window function). The points indicate an extraction area in $\mathrm{Mpc}^{2}$ as a function of the radius of the zone, calculated as $\left(r_{\min }+r_{\max }\right) / 2$. The grey lines indicate a quadratic dependence of the area on the radius. The data is in remarkably good agreement with these curves, indicating that the sampling corresponds roughly to a grid in cylindrical coordinates. The upper dashed line approximately corresponds to the case of no sampling on azimuth, solid line to having 3 regions, and low dashed line: 10 to sample the azimuthal scale.

the azimuthal angle with typically 3 sectors on radial scales from 0.1 to $1 \mathrm{Mpc}$.

Since there is some discussion on the reliability of the temperature determination, we have provided a plot for each cluster, where a comparison to the average cluster temperature profile of Vikhlinin et al. (2005) is presented. One can see in each case that there is good agreement in the results. We also point out that a few clusters where we probed the region outside $r_{500}$ reveal strong asymmetries that indicate accretion from a filament. It may be that the presence or removal of such zones could be the underlying reason for some of the reported disagreement in temperature profiles.

We define a cool core of a cluster or its debris as the gas with entropy significantly below $200-300 \mathrm{keV} \mathrm{cm}^{2}$, which according to Voit \& Bryan (2001) could cool in a Hubble time. As local examples of cool cores have entropies lower than

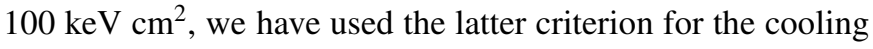
core identification.

Before proceeding with the description of individual systems, we summarize the results by presenting the fits to the entropy and pressure profiles. To define the shape of the entropy and pressure profiles, we applied the non-parametric locally weighted regression, following Sanderson et al. (2005, and references therein). This analysis results in the non-parametric curve, which we approximate below with power laws. Our analysis, illustrated in Fig. 2, suggests a broken power law approximation to the entropy profile with inner and outer slopes of 0.78 and 0.52 , respectively, and a break at $0.5 r_{500}$. The amplitude of fluctuations around the best exceeds the effect of the 

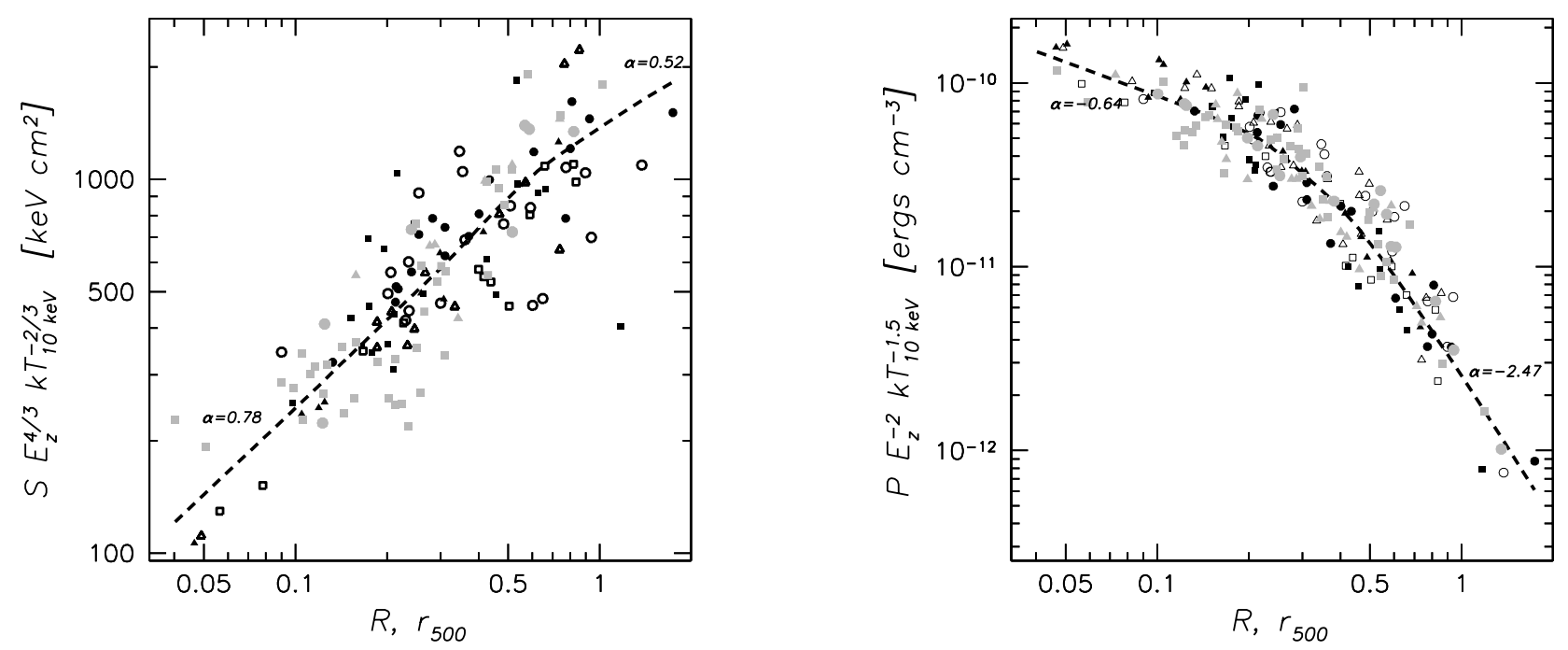

Fig. 2. Comparison between the entropy and pressure of the sample and the analytical approximation, used to study the dispersion. The entropy and pressure points corresponding to the same cluster are shown using the same symbol. The dashed line shows the results of the fit using the non-parametric locally weighted regression method.

statistics and is a measure of the importance of substructure, as discussed below. The average level of fluctuations, which is $15 \%(20 \%)$ in the case of entropy (pressure), could be taken as the accuracy to which the approximation to the entropy distribution could be determined. The entropy profiles with exclusion of the substructure has been analyzed in Zhang et al. (2005) to yield a steeper index of 0.95. Charactering the pressure profile is more complex, requiring three power laws, with slopes -0.64 at $r<0.3 r_{500},-2.47$ at $r>0.5 r_{500}$ and a slope of -1.50 in between. We note here, that the outer pressure slope of -2.47 could be set in correspondence to the $3 \beta \gamma$ term in the mass estimation based on the hydrostatic equilibrium. For a typical value of $\gamma=1.2$ (Finoguenov et al. 2001), we obtain a representative value of $\beta=0.69$, which is consistent with the values used in Finoguenov et al. (2001). For completeness, in Table 2 we present the standard approach of using the orthogonal regression and assuming a power law shape to approximate the shape of the entropy and pressure profiles. We present the results obtained using different masks and also combine the clusters with and without the rescaling, described above. As could be easily seen from Table 3, this approach results in a much larger amplitude of the residuals, even taking into account that the power law shape was fitted separately for each cluster, while in the non-parametric approach, one shape is used to approximate all clusters.

One of the most important results is an observation of a flattening in the entropy profile at outer radii in DXL clusters, changing from 0.78 within the $0.5 r_{500}$ to 0.54 outside. As the sample consists of the most massive clusters in the Universe, we believe that the explanation of the observed trend should be searched in the details of the accretion. As summarized in Voit (2005), the index of the entropy profile is driven by the effects of mass growth, as well as evolution of the virial density. Under the assumption of a smooth accretion, the entropy grows with radius as $S \sim M_{\text {gas }}^{1-4 / 3}$ (Voit 2005). With a canonical cluster characteristic of the surface brightness profile, $\beta=2 / 3$, $M_{\text {gas }} \sim r$, where $M_{\text {gas }}$ is enclosed gas mass. However, as gas
Table 2. Results of the orthogonal regression analysis of entropy and pressure profiles.

\begin{tabular}{|c|c|c|c|c|}
\hline \multirow{2}{*}{$\begin{array}{l}\text { Name } \\
\text { RXCJ }\end{array}$} & \multirow{2}{*}{ 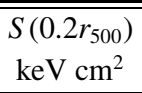 } & $S$ slope & $P\left(0.2 r_{500}\right)$ & $P$ slope \\
\hline & & \multicolumn{3}{|c|}{$10^{-11} \mathrm{erg} \mathrm{cm}^{-3}$} \\
\hline \multirow[t]{2}{*}{$0014.3-3022$} & $373 \pm 21$ & $0.30 \pm 0.12$ & $7.8 \pm 1.1$ & $-1.70 \pm 0.34$ \\
\hline & $352 \pm 29$ & $0.40 \pm 0.13$ & $13.9 \pm 1.6$ & $-2.28 \pm 0.23$ \\
\hline \multirow[t]{2}{*}{ 0043.4-2037 } & $416 \pm 32$ & $0.05 \pm 0.30$ & $5.2 \pm 0.7$ & $-1.69 \pm 0.21$ \\
\hline & $287 \pm 17$ & $0.61 \pm 0.16$ & $5.1 \pm 0.3$ & $-1.92 \pm 0.12$ \\
\hline \multirow[t]{2}{*}{$0232.2-4420$} & $280 \pm 18$ & $0.60 \pm 0.19$ & $4.3 \pm 0.4$ & $-1.35 \pm 0.16$ \\
\hline & $236 \pm 46$ & $0.50 \pm 0.62$ & $4.2 \pm 0.6$ & $-0.91 \pm 0.40$ \\
\hline \multirow[t]{2}{*}{ 0307.0-2840 } & $222 \pm 7$ & $0.91 \pm 0.12$ & $2.8 \pm 0.2$ & $-1.41 \pm 0.12$ \\
\hline & $243 \pm 3$ & $0.93 \pm 0.06$ & $3.3 \pm 0.2$ & $-1.35 \pm 0.20$ \\
\hline \multirow[t]{2}{*}{ 0528.9-3927 } & $173 \pm 17$ & $0.86 \pm 0.21$ & $4.4 \pm 0.4$ & $-1.55 \pm 0.20$ \\
\hline & $124 \pm 24$ & $1.10 \pm 0.36$ & $4.9 \pm 0.5$ & $-1.64 \pm 0.43$ \\
\hline \multirow[t]{2}{*}{ 1131.9-1955 } & $279 \pm 22$ & $0.73 \pm 0.24$ & $6.2 \pm 0.7$ & $-1.72 \pm 0.24$ \\
\hline & $238 \pm 10$ & $0.79 \pm 0.18$ & $5.9 \pm 0.5$ & $-1.83 \pm 0.21$ \\
\hline \multirow[t]{2}{*}{$2337.6+0016$} & $324 \pm 20$ & $0.80 \pm 0.15$ & $3.9 \pm 0.2$ & $-1.20 \pm 0.15$ \\
\hline & $354 \pm 35$ & $0.63 \pm 0.30$ & $4.3 \pm 0.7$ & $-1.39 \pm 0.48$ \\
\hline \multirow[t]{2}{*}{ 0532.9-3701 } & $435 \pm 33$ & $0.11 \pm 0.10$ & $2.8 \pm 0.7$ & $-0.64 \pm 0.52$ \\
\hline & $296 \pm 13$ & $0.21 \pm 0.23$ & $6.8 \pm 0.3$ & $-2.18 \pm 0.13$ \\
\hline \multirow[t]{2}{*}{$0658-5557$} & $382 \pm 15$ & $0.86 \pm 0.11$ & $8.3 \pm 0.5$ & $-1.34 \pm 0.21$ \\
\hline & $379 \pm 16$ & $0.94 \pm 0.09$ & $7.5 \pm 0.4$ & $-1.62 \pm 0.17$ \\
\hline all & $290 \pm 8$ & $0.62 \pm 0.09$ & $5.4 \pm 0.2$ & $-1.54 \pm 0.10$ \\
\hline$r>0.25 r_{500}$ & $381 \pm 53$ & $0.38 \pm 0.27$ & $11.4 \pm 1.0$ & $-2.15 \pm 0.17$ \\
\hline$r<0.25 r_{500}$ & $388 \pm 31$ & $1.33 \pm 0.37$ & $6.0 \pm 0.5$ & $-0.77 \pm 0.44$ \\
\hline$T \& z$-corrected & $452 \pm 11$ & $0.64 \pm 0.08$ & $6.8 \pm 0.2$ & $-1.43 \pm 0.10$ \\
\hline$r>0.25 r_{500}$ & $570 \pm 72$ & $0.44 \pm 0.24$ & $11.5 \pm 1.1$ & $-1.88 \pm 0.18$ \\
\hline$r<0.25 r_{500}$ & $509 \pm 31$ & $1.10 \pm 0.27$ & $5.8 \pm 0.5$ & $-1.09 \pm 0.45$ \\
\hline
\end{tabular}

mass fraction tends to level off at high radii, the cumulative gas mass starts to follow the mass of the dark matter and so $M_{\text {gas }} \sim r^{0.5}$. A similar flattening in the entropy distribution is then expected and is observed in our data at $r \geq 0.4 r_{500}$. If this is indeed the explanation of our data, one would not necessarily expect the same trend to be observed in low-mass 
Table 3. Entropy and pressure fluctuations around the power law approximation, reported in Table 2.

\begin{tabular}{rcccccc}
\hline \hline Name & $\sigma S$ & $\sigma P$ & $\sigma S$ & $\sigma P$ & \multicolumn{2}{c}{$\sigma S$} \\
RXCJ & \multicolumn{2}{c}{$r<0.3 r_{500}$} & \multicolumn{2}{c}{$r>0.2 r_{500}$} & \multicolumn{2}{c}{$\sigma P$} \\
from individual fits \\
\hline $0014.3-3022$ & $0.58 \pm 0.07$ & $0.62 \pm 0.07$ & $0.26 \pm 0.07$ & $0.45 \pm 0.05$ & $0.26 \pm 0.07$ & $0.75 \pm 0.08$ \\
& $0.47 \pm 0.07$ & $0.74 \pm 0.06$ & $0.10 \pm 0.09$ & $0.47 \pm 0.07$ & $0.12 \pm 0.05$ & $1.05 \pm 0.08$ \\
$0043.4-2037$ & $0.49 \pm 0.06$ & $0.64 \pm 0.11$ & $0.44 \pm 0.12$ & $0.44 \pm 0.20$ & $0.47 \pm 0.14$ & $1.04 \pm 0.14$ \\
& $0.44 \pm 0.11$ & $0.64 \pm 0.09$ & $0.08 \pm 0.08$ & $0.11 \pm 0.08$ & $3.61 \pm 0.25$ & $0.91 \pm 0.42$ \\
$0232.2-4420$ & $0.34 \pm 0.16$ & $0.52 \pm 0.06$ & $0.46 \pm 0.21$ & $0.47 \pm 0.16$ & $0.40 \pm 0.20$ & $0.58 \pm 0.05$ \\
& $0.21 \pm 0.04$ & $0.62 \pm 0.15$ & $0.25 \pm 0.05$ & $0.41 \pm 0.16$ & $0.25 \pm 0.05$ & $1.00 \pm 0.11$ \\
$0307.0-2840$ & $0.29 \pm 0.06$ & $0.28 \pm 0.05$ & $0.26 \pm 0.07$ & $0.24 \pm 0.07$ & $0.28 \pm 0.06$ & $0.25 \pm 0.06$ \\
& $0.10 \pm 0.03$ & $0.45 \pm 0.05$ & $0.16 \pm 0.04$ & $0.17 \pm 0.04$ & $0.04 \pm 0.02$ & $0.48 \pm 0.05$ \\
$0528.9-3927$ & $0.28 \pm 0.12$ & $0.46 \pm 0.08$ & $0.22 \pm 0.11$ & $0.36 \pm 0.13$ & $0.33 \pm 0.11$ & $0.39 \pm 0.10$ \\
& $0.16 \pm 0.06$ & $0.67 \pm 0.08$ & $0.14 \pm 0.10$ & $0.40 \pm 0.09$ & $0.17 \pm 0.06$ & $0.53 \pm 0.09$ \\
$1131.9-1955$ & $0.42 \pm 0.05$ & $0.45 \pm 0.07$ & $0.29 \pm 0.05$ & $0.32 \pm 0.06$ & $0.37 \pm 0.04$ & $0.50 \pm 0.07$ \\
& $0.57 \pm 0.05$ & $0.74 \pm 0.08$ & $0.29 \pm 0.07$ & $0.44 \pm 0.08$ & $0.78 \pm 0.05$ & $0.81 \pm 0.07$ \\
$2337.6+0016$ & $0.31 \pm 0.08$ & $0.44 \pm 0.18$ & $0.26 \pm 0.08$ & $0.38 \pm 0.20$ & $0.23 \pm 0.09$ & $0.49 \pm 0.16$ \\
& $0.22 \pm 0.05$ & $0.41 \pm 0.11$ & $0.15 \pm 0.05$ & $0.23 \pm 0.16$ & $0.15 \pm 0.05$ & $0.54 \pm 0.09$ \\
$0532.9-3701$ & $0.58 \pm 0.34$ & $0.75 \pm 0.52$ & $0.43 \pm 0.33$ & $0.44 \pm 0.30$ & $0.41 \pm 0.31$ & $1.41 \pm 0.72$ \\
& $0.52 \pm 0.05$ & $0.73 \pm 0.09$ & $0.35 \pm 0.06$ & $0.28 \pm 0.07$ & $0.36 \pm 0.05$ & $0.62 \pm 0.08$ \\
$0658-5557$ & $0.27 \pm 0.05$ & $0.54 \pm 0.07$ & $0.25 \pm 0.07$ & $0.40 \pm 0.11$ & $0.19 \pm 0.07$ & $0.57 \pm 0.07$ \\
& $0.18 \pm 0.04$ & $0.62 \pm 0.06$ & $0.21 \pm 0.06$ & $0.25 \pm 0.04$ & $0.13 \pm 0.04$ & $0.55 \pm 0.05$ \\
\hline
\end{tabular}

Table 4. Entropy and pressure fluctuations around the mean sample trend. Results of the spectral analysis using two different masks are shown. The $I-T$ is the mask based on cross-sections of the isothermal and isodensity regions. The $S-P$ is the mask based on the cross-sections of the isentropic and isobaric regions.

\begin{tabular}{|c|c|c|c|c|}
\hline \multirow{2}{*}{$\begin{array}{l}\text { Name } \\
\text { RXCJ }\end{array}$} & $\sigma S$ & $\sigma P$ & $\sigma S$ & $\sigma P$ \\
\hline & \multicolumn{2}{|c|}{$I-T$-mask } & \multicolumn{2}{|c|}{$S$-P-mask } \\
\hline $0014.3-3022$ & $0.22 \pm 0.06$ & $0.25 \pm 0.05$ & $0.15 \pm 0.06$ & $0.31 \pm 0.09$ \\
\hline 0043.4-2037 & $0.18 \pm 0.13$ & $0.33 \pm 0.25$ & $0.04 \pm 0.04$ & $0.26 \pm 0.25$ \\
\hline $0232.2-4420$ & $0.42 \pm 0.20$ & $0.42 \pm 0.20$ & $0.15 \pm 0.05$ & $0.30 \pm 0.13$ \\
\hline 0307.0-2840 & $0.20 \pm 0.06$ & $0.17 \pm 0.06$ & $0.09 \pm 0.05$ & $0.10 \pm 0.05$ \\
\hline 0528.9-3927 & $0.16 \pm 0.05$ & $0.20 \pm 0.13$ & $0.12 \pm 0.09$ & $0.29 \pm 0.08$ \\
\hline 1131.9-1955 & $0.12 \pm 0.04$ & $0.13 \pm 0.05$ & $0.28 \pm 0.07$ & $0.26 \pm 0.08$ \\
\hline $2337.6+0016$ & $0.22 \pm 0.07$ & $0.33 \pm 0.17$ & $0.10 \pm 0.03$ & $0.18 \pm 0.13$ \\
\hline 0532.9-3701 & $0.41 \pm 0.34$ & $0.41 \pm 0.34$ & $0.35 \pm 0.05$ & $0.25 \pm 0.07$ \\
\hline $0658-5557$ & $0.24 \pm 0.10$ & $0.35 \pm 0.13$ & $0.15 \pm 0.05$ & $0.25 \pm 0.11$ \\
\hline
\end{tabular}

clusters, where the baryon fraction is growing with the radius even at $r_{500}$.

In addition to this explanation, we would like to mention that with faster mass growth of the cluster (as during a major merger), the entropy profile should become flatter (Voit 2005). Yet another effect, associated with the survival of the low entropy gas during the merger (Motl et al. 2004), reduces the increase in the entropy associated with the mass growth and should effectively lead to flatter entropy profiles; however, full details should be obtained from simulations.

Once the general behavior of the entropy and pressure was found, we studied the amplitude of the deviations from the average profile, allowing for the change in the normalization, and then compared that with the statistical errors in Table 4.

In Fig. 3 we study the scatter in the entropy and pressure, reported in Table 4. The dispersion and normalization of the profile are calculated weighting the points by the area of the corresponding region. The renormalization is needed to remove a possible bias due to assumption of a mean temperature in

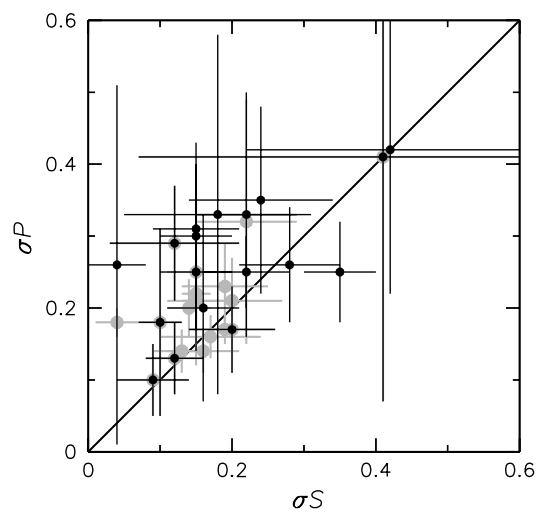

Fig. 3. Correlation in the dispersion of entropy and pressure. Grey points indicate measurements within $0.4 r_{500}$ and solid points - in full range of radii. The solid line shows the one-to-one ratio between the entropy and pressure.

rescaling the profiles. We note that the spread of values for the normalization is larger for the pressure, where the sensitivity to 

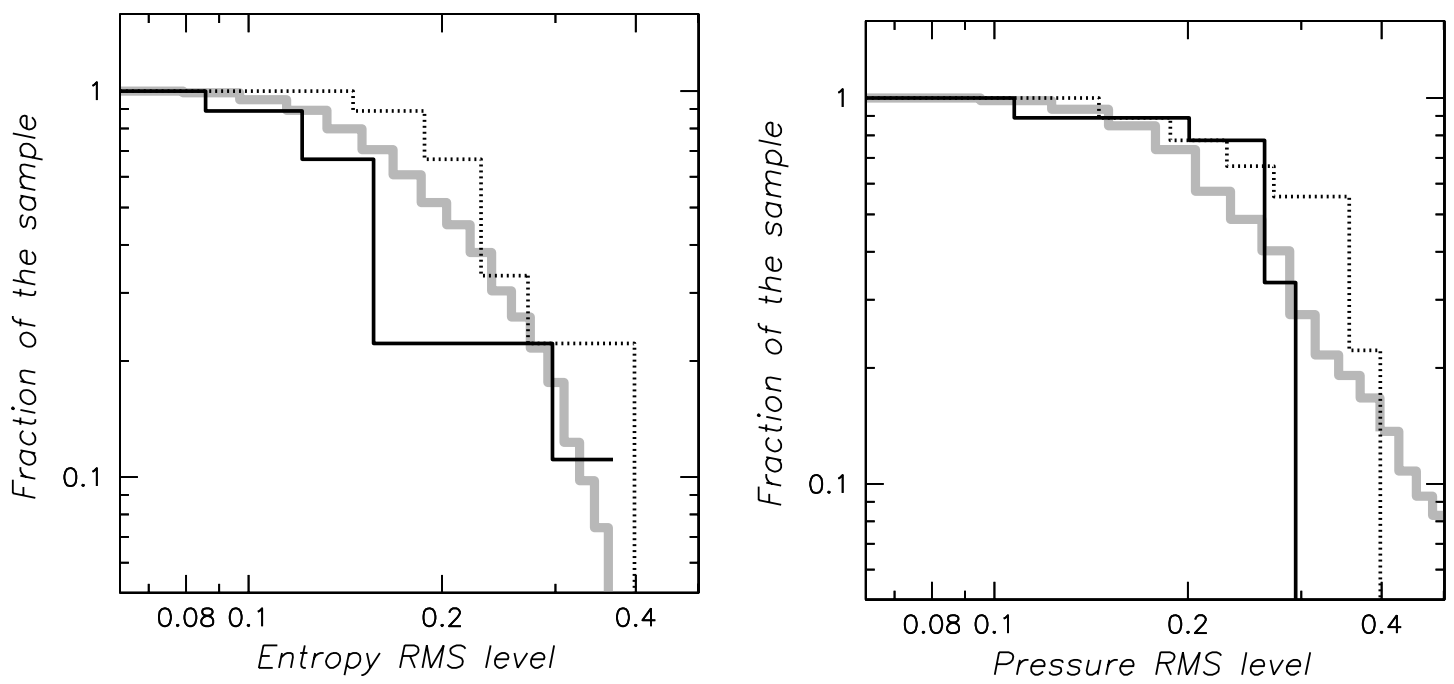

Fig. 4. Fraction of clusters with rms of the entropy (left panel) and pressure (right panel) parameter greater than the $x$-axis value. Black lines denote the results for DXL cluster sample, obtained using two different masks (marked as solid and dotted lines), correspondingly sampling entropy/pressure and image/temperature. The grey line represents the results of a similar analysis performed on a sample of 208 modeled clusters (Finoguenov et al. 2005).

the assumed weighted temperature is also larger. There is also no clear correlation between the r.m.s. values and the normalization in either entropy or pressure. The figure shows that the fluctuations in the entropy and pressure are correlated in the magnitude, with the relation favoring somewhat higher pressure fluctuations as compared to the entropy, but certainly not following the prescription for the shock heating, which at the observed amplitudes predicts fluctuations only in the pressure and very little in the entropy. This picture and a quantitative analysis below is very similar to the results of Finoguenov et al. (2005), who have analyzed a set of cosmological simulations of clusters. Apparently, azimuthal distortions, related to gas and dark matter displacements during the merger and post relaxation, supersede the distortions associated with the shock heating. The latter accounts for about $15 \%$, and its clear separation could be better revealed in the future by comparing pressure maps derived at X-rays and thermal SZE observations.

In Fig. 4 we summarize the fluctuation analysis and compare it to the distribution for the cluster sample obtained in the cosmological simulations (Finoguenov et al. 2005), which samples a similar range of radii. Taking the comparison between the model and the results obtained with the mask sampling pressure and entropy, we conclude that the clusters show a similar degree of fluctuations and the probability of parameters of our sample being randomly drawn from the same distribution, calculated using the KS-test, is $60 \%$ and $80 \%$ for the pressure and entropy, respectively. We combined the results of the two masks in the test.

\subsection{RXCJ0014.3-3022}

The cluster consists of two clumps. The large-scale emission is centered on one of the peaks, which is therefore taken for the center of the main cluster, and its position is listed in Table 1. In order to tabulate the basic properties of the cluster, we extracted the spectra from zones that according to the hardness ratio have the same temperature to within $1 \mathrm{keV}$. Table 5 contains three zones with temperatures exceeding $7 \mathrm{keV}$. These zones form a ridge passing between the two subclusters and surrounding the main cluster center. The elongated shape of the ridge suggests its origin in the interaction between the clusters. The temperature enhancement compared to region 5 is a factor of $1.4 \pm 0.1$, which corresponds to a Mach number of $1.4 \pm 0.1$. Although higher temperatures are also observed, they are not statistically different from $9 \mathrm{keV}$. The pressure peak is not at the cluster center, but is within the ridge.

The temperature, as well as the pressure of the subcluster (region 8), are similar to that of the disrupted core of the main cluster (region 7, as well as 5). Assuming that the mass scales with the central pressure as $M \sim P^{1.5}$, the initial mass ratio of the two clusters is $1.7 \pm 0.2$. An expected final temperature of the cluster after the merger from the $M-T$ relation (Finoguenov et al. 2001) is $9.1 \pm 0.5 \mathrm{keV}$, similar to the observed temperature of the ridge. This comparison suggests that the grown mass of the new cluster will be able to cope with the increased pressure of the gas.

In Fig. 5 we also compare the derived entropies and pressures with the average trend for the DXL sample and further illustrate their ratio in Figs. 14 and 15. It can be seen that the entropy of the core region is higher by a factor of $1.3 \pm 0.05$. Low entropy clump is seen at the position of the second core. The pressure of the bridge appears to match the adopted scaling temperature of $8.3 \mathrm{keV}$ well. The pressure of the main cluster at the distance of the subcluster (region 5) is higher by a factor of $1.5 \pm 0.05$ than the model, despite a relatively low temperature of $6.7 \mathrm{keV}$.

The subcluster is characterized by higher pressure (best seen in the Table 5 and as a deviation in the pressure profile), as well as lower entropy. This implies that the low entropy gas of the subcluster is retained within its own dark matter halo. This picture is different from the case of A3667 (Briel et al. 2003), where low entropy gas is displaced from the pressure 

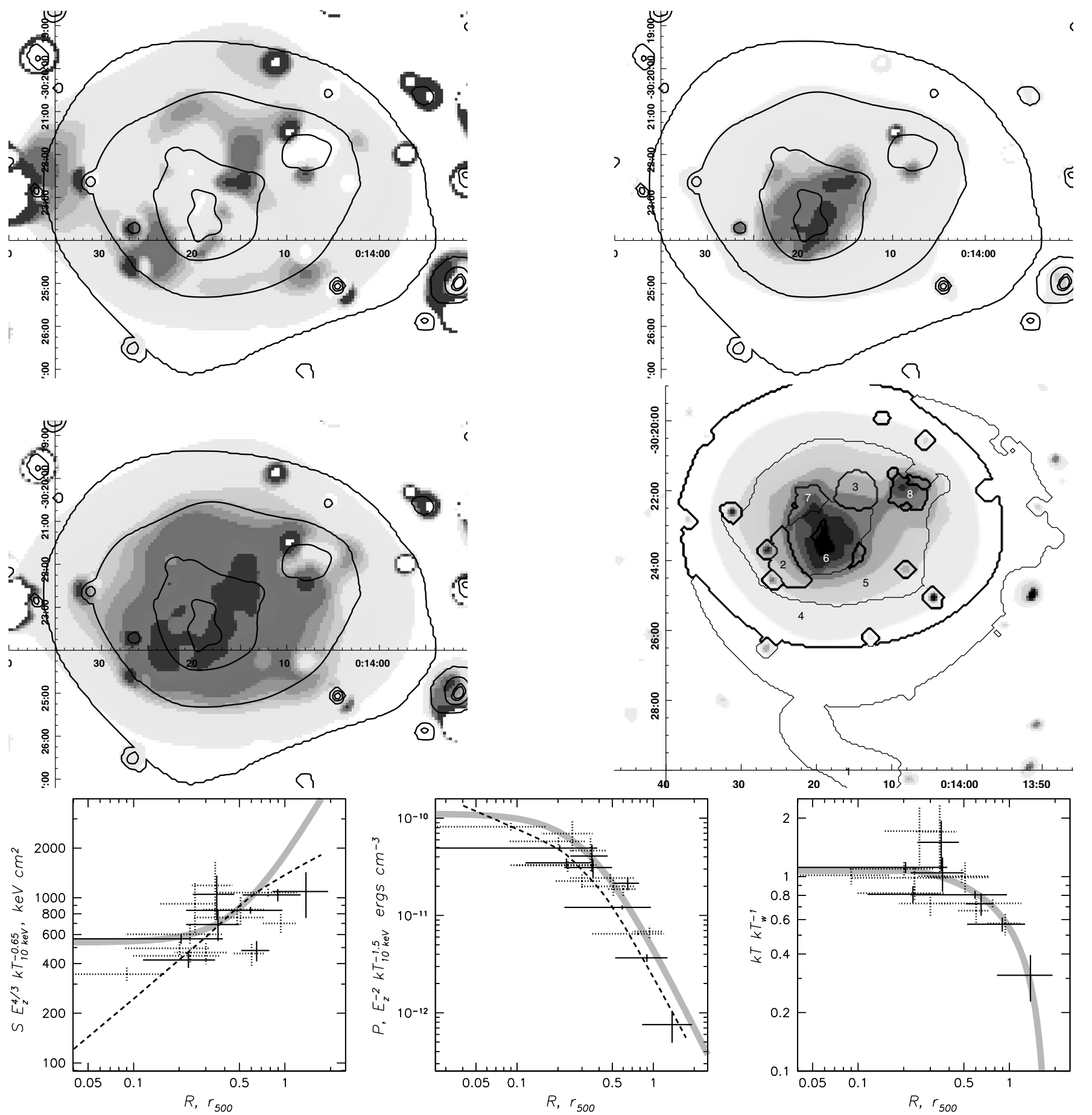

Fig. 5. RXCJ0014.3-3022. Top left: entropy map; top right: pressure map; middle left: temperature map; middle right: surface brightness. Entropy, pressure, and temperature maps are overlaid with the contours of equal surface brightness in the $0.5-2 \mathrm{keV}$ band. The surface brightness image is overlaid with contours showing the spectral extraction regions with numbers corresponding to those in Table 5. Coordinate grids are shown for the epoch J2000. On the corresponding maps, zones of low entropy are shown in white, zones of higher pressure, temperature are shown in black. Lower left: entropy profile, lower center: pressure profile, lower right: temperature profile. The solid crosses denotes the tabulated data and the dotted crosses show the rest of the results. The data is scaled for both the cluster mass, using the relation of Ponman et al. (2003) and evolution of the critical density with redshift. Dashed lines on both plots shows the best fit to the whole sample, described in the text. Grey lines show the results of the 1d analysis, using a beta-model and fits to the temperature profile from Zhang et al. (2005, in prep.). The grey line in the temperature panel is the universal temperature profile of Vikhlinin et al. (2005).

peak. The subcluster in A2744 is a cool core, rather than a cold front. Our identification of the core of the main cluster is further confirmed by the entropy plot, as it lies on the prediction for the self-similar scaling. It is located $0.2 \mathrm{Mpc}$ apart from the cluster center and may even present the still unperturbed part of an initially large cluster core extending to that radii. 
Table 5. Properties of the main regions of RXCJ0014.3-3022.

\begin{tabular}{ccccccccc}
\hline \hline$N$ & $\begin{array}{c}k T \\
\mathrm{keV}\end{array}$ & $\begin{array}{c}\rho_{e} \\
10^{-4} \mathrm{~cm}^{-3}\end{array}$ & $\begin{array}{c}S \\
\mathrm{keV} \mathrm{cm}^{2}\end{array}$ & $\begin{array}{c}P, 10^{-12} \\
\mathrm{ergs} \mathrm{cm}^{-3}\end{array}$ & $\begin{array}{c}M_{\text {gas }} \\
10^{12} M_{\odot}\end{array}$ & $\begin{array}{c}r_{\min } \\
\mathrm{Mpc}\end{array}$ & $\begin{array}{c}r_{\max } \\
\mathrm{Mpc}\end{array}$ & Remarks \\
\hline 1 & $2.6 \pm 0.7$ & $1.9 \pm 0.4$ & $785 \pm 242$ & $0.8 \pm 0.3$ & $25.9 \pm 5.9$ & 0.92 & 2.14 & main-3 \\
2 & $12.5 \pm 3.6$ & $21.2 \pm 2.3$ & $754 \pm 226$ & $42.4 \pm 13.2$ & $2.7 \pm 0.3$ & 0.27 & 0.51 & ridge S \\
3 & $8.7 \pm 1.7$ & $23.2 \pm 1.6$ & $495 \pm 102$ & $32.2 \pm 6.8$ & $3.4 \pm 0.2$ & 0.25 & 0.56 & ridge N \\
4 & $4.7 \pm 0.4$ & $5.0 \pm 0.2$ & $748 \pm 64$ & $3.8 \pm 0.3$ & $58.8 \pm 1.8$ & 0.58 & 1.41 & main-2 \\
5 & $6.7 \pm 0.3$ & $11.7 \pm 0.2$ & $604 \pm 29$ & $12.5 \pm 0.6$ & $49.1 \pm 0.7$ & 0.25 & 1.07 & main-1 \\
6 & $9.2 \pm 0.6$ & $34.5 \pm 0.6$ & $405 \pm 27$ & $51.1 \pm 3.4$ & $14.9 \pm 0.2$ & 0.03 & 0.43 & ridge \\
7 & $6.7 \pm 0.7$ & $33.3 \pm 1.6$ & $302 \pm 32$ & $35.9 \pm 4.0$ & $2.7 \pm 0.1$ & 0.13 & 0.38 & disrupted core \\
8 & $6.0 \pm 0.8$ & $23.0 \pm 1.6$ & $345 \pm 49$ & $22.2 \pm 3.3$ & $3.2 \pm 0.2$ & 0.57 & 0.87 & subcluster \\
\hline
\end{tabular}

Table 6. Properties of the main regions of RXCJ0043.4-2037.

\begin{tabular}{ccccccccc}
\hline \hline$N$ & $\begin{array}{c}k T \\
\mathrm{keV}\end{array}$ & $\begin{array}{c}\rho_{e} \\
10^{-4} \mathrm{~cm}^{-3}\end{array}$ & $\begin{array}{c}S \\
\mathrm{keV} \mathrm{cm}^{2}\end{array}$ & $\begin{array}{c}P, 10^{-12} \\
\mathrm{ergs} \mathrm{cm}\end{array}$ & $\begin{array}{c}M_{\text {gas }} \\
10^{12} M_{\odot}\end{array}$ & $\begin{array}{c}r_{\min } \\
\mathrm{Mpc}\end{array}$ & $\begin{array}{c}r_{\max } \\
\mathrm{Mpc}\end{array}$ & Remarks \\
\hline 1 & $7.0 \pm 0.6$ & $36.0 \pm 0.8$ & $300 \pm 24$ & $40.6 \pm 3.4$ & $9.7 \pm 0.2$ & 0.04 & 0.40 & core \\
2 & $7.2 \pm 1.1$ & $18.7 \pm 1.3$ & $475 \pm 78$ & $21.6 \pm 3.7$ & $3.9 \pm 0.3$ & 0.10 & 0.53 & \\
3 & $5.1 \pm 0.4$ & $12.2 \pm 0.5$ & $450 \pm 40$ & $10.1 \pm 1.0$ & $16.0 \pm 0.7$ & 0.11 & 0.64 & \\
4 & $6.0 \pm 0.6$ & $21.5 \pm 1.0$ & $361 \pm 38$ & $20.7 \pm 2.3$ & $7.0 \pm 0.3$ & 0.00 & 0.49 & \\
5 & $2.7 \pm 1.0$ & $1.5 \pm 0.4$ & $963 \pm 379$ & $0.7 \pm 0.3$ & $37.6 \pm 9.0$ & 0.81 & 2.71 & filament? \\
6 & $4.5 \pm 0.7$ & $4.5 \pm 0.4$ & $772 \pm 119$ & $3.2 \pm 0.5$ & $27.7 \pm 2.2$ & 0.51 & 1.11 & outskirts \\
\hline
\end{tabular}

In Fig. 5 we also show the results produced by the standard analysis using the beta model (e.g. Jones \& Forman 1984). We find an overall agreement between the two methods.

For many of the described features there is a qualitative agreement with Chandra results of Kempner \& David (2004). Similar high temperature ridges have been claimed for A1644 (Reiprich et al. 2004) and A3921 (Belsole et al. 2005), yet none of them are found to dominate the pressure of the cluster, as in the case of A2744.

\subsection{RXCJ0043.4-2037}

Judging from the image, this is one of the most relaxed clusters in the sample. The image has a single center, which is located at the center of the large-scale emission. However, there is an indication of small pressure and entropy distortions and the statistical analysis reveals that the fluctuations are quite large. The pressure shape essentially follows the temperature shape. Some small-scale fluctuations in the temperature map are still seen. The image is extended to the north-west.

The results of the spectral analysis are reported in Table 6 and shown in Fig. 6. The most significant feature is the presence of a region with extremely low entropy, seen as a dotted cross in the entropy profile in Fig. 6 at $0.7 r_{500}$ distance to the center to the south. This gas is in pressure equilibrium with the cluster, suggesting that we observe the debris of an accreted group. Comparison with simulations would be useful here to shed more light on the stage of this suggested disruption. Given the statistical uncertainty, it is difficult to conclude much about the origin of the extent towards the north-west in the lowest levels of the X-ray surface brightness (region 5). Presumably, this extension, located outside of $r_{500}$, is due to an accretion of a filament.

\section{3. $R X C J 0232.2-4420$}

The hardness ratio map reveals a soft compact source in the center. In the image we see some elongation to the north. In the inner region, the surface brightness distribution indicates a triangular shape with one tip of the triangle pointing towards the north and the other to the west. As a result what we see is that there is colder material in the east compared to the west. The pressure map looks rather symmetric, but we have an asymmetric entropy structure. While a possible scenario could be a slow infall of the material from the north-east, the west part of the cluster appears to be systematically hotter as compared to the eastern side, which could indicate a strong shock.

The spectroscopic analysis is reported in Table 7 and Fig. 7. The point-like source appears to be the low-entropy core of the cluster, with one of the lowest entropies $\left(77 \pm 4 \mathrm{keV} \mathrm{cm}^{2}\right)$ in our sample. The hot region on the west is characterized by both an enhancement in the pressure and entropy, yet of high statistical uncertainty. We attribute the appearance of this region to a forward shock of the Mach number of $3 \pm 1$. The low entropy gas in the north east (region 9) is confirmed in the spectral analysis. The behavior of the entropy in the outskirts of this cluster led us to conclude, supported by other clusters in this sample, that the clusters in the advanced stage of interaction have systematically higher entropy at $r_{500}$ compared to the average trend. We ascribe this result to the heating by the forward shock, induced during the merger.

\subsection{RXCJ0307.0-2840}

This cluster seems to be quite relaxed judging from the symmetric appearance of the image and the pressure map. The temperature map shows small amplitude fluctuations. According to 

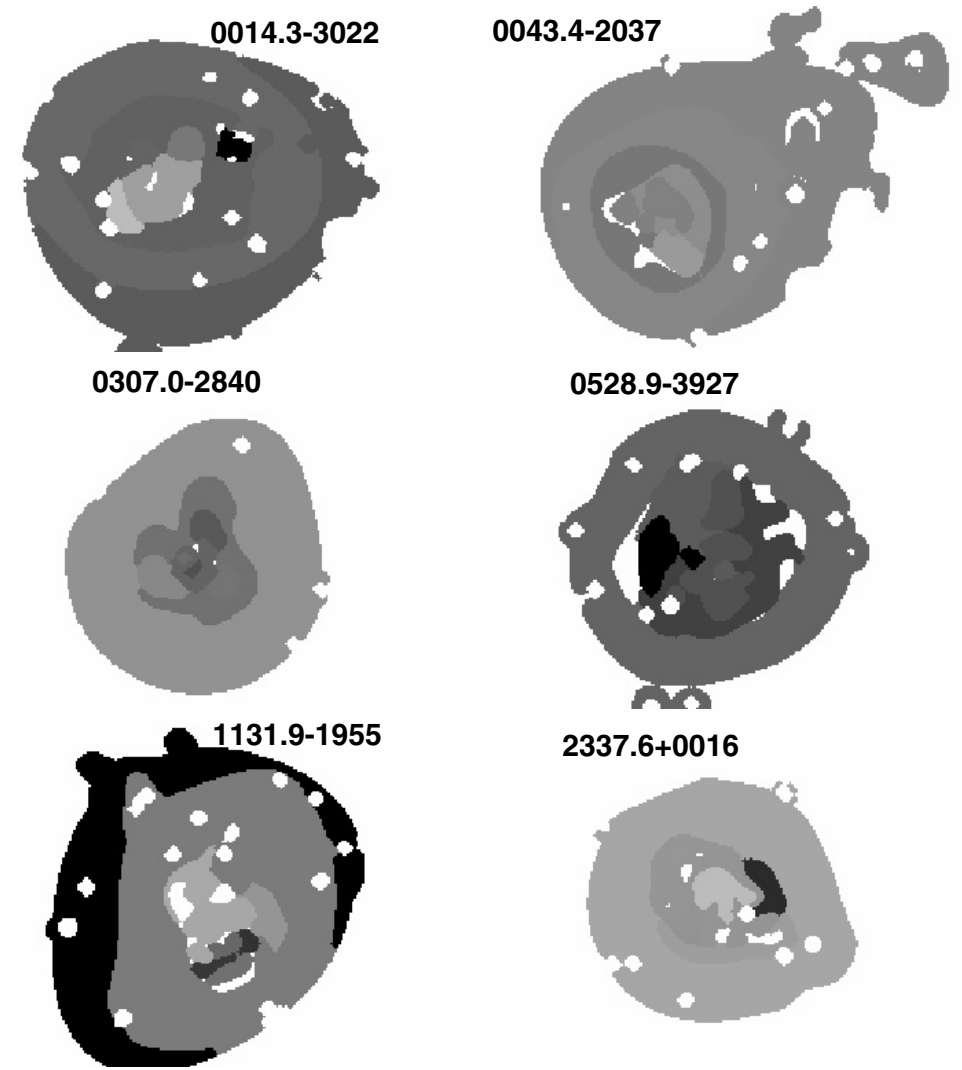

\section{9-3927}

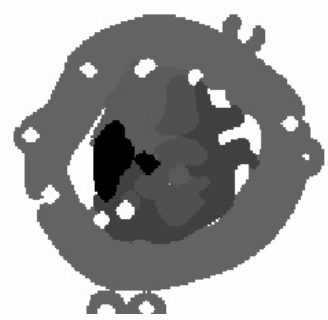

$2337.6+0016$

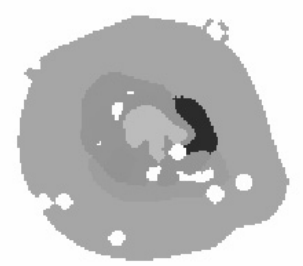

0232.2-4420

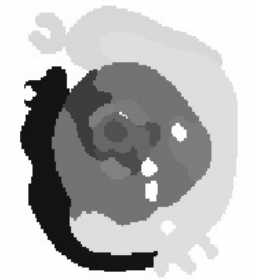

0532.9-3701

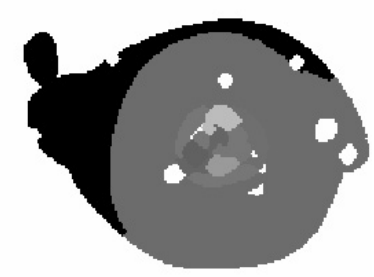

0658-5557

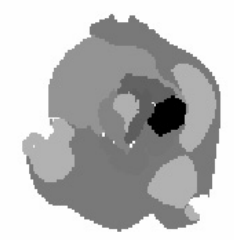

Fig. 14. Maps of the ratio between the observed entropy and the average trends measured in the DXL sample. The different shades of grey stand for a different value of the ratio, light - 1.9 (seen only in RXCJ 0232.2-4420 cluster), grey - 1.1 (a dominant color of both RXCJ 0043.4-2037 and RXCJ 0307.0-2840 clusters), dark grey - 0.9 (a dominant color of RXCJ 0014.3-3022), black - 0.4 (e.g. tail of RXCJ 0532.9-3701).

the spectroscopic analysis, reported in Table 8 and shown in Fig. 8, the cluster has a cool core, but otherwise exhibits no significant deviations from the modified scaling relation and bears a slightly higher entropy and shows no fluctuations in the pressure profile. The $1 \mathrm{~d}$ and $2 \mathrm{~d}$ modeling agree well with each other.

\subsection{RXCJ0528.9-3927}

A strong soft point source near the center ( $<20 \%$ of the flux) prevents a more detailed analysis of the central region. The pressure peak is distorted on small scales, while on large scales it appears quite relaxed. There is some entropy and temperature structure in the north-west.

The spectroscopic analysis reported in Table 9 and Fig. 9 does not include region 1, which is associated with the pointlike source and reveals a clearly non-thermal spectrum. The suggested fluctuations in the north-west are confirmed as a zone of lower entropy, and also in general the entropy of this cluster is lower at almost every radii compared to the general trend.

\subsection{RXCJ0532.9-3701}

This cluster shows one of the most symmetrical images in the sample. It has a low-entropy core, but not low enough for a cool core. The pressure map is also quite symmetric. In the intensity, we see a slightly boxy structure and associate it with a clover leaf structure in the entropy. On large scales the temperature is anti-correlated to the density in azimuthal direction, resulting in a constant pressure.

The spectroscopic analysis is reported in Table 10 and Fig. 10. This cluster exhibits a low-entropy infalling zone to the east (region 8). Its entropy is $286 \pm 65 \mathrm{keV} \mathrm{cm}^{2}$, which is lower than the $665 \pm 55 \mathrm{keV} \mathrm{cm}^{2}$ value for the preceding zone (region 9). However, the low-entropy zone exhibits a pressure typical of its distance to the center. The indicated pressure and entropy fluctuations are rather marginal, $\sim 2$ sigma. The strong fluctuations in both entropy and pressure, reported in Table 4, are entirely due to the presence of the filament.

\subsection{RXCJ1131.9-1955 ("whirlpool", A1300)}

The most remarkable feature of this cluster is an unusually elongated pressure map. We coined the name "whirlpool" cluster for this object due to the observed propeller-like temperature structure. Three temperature rims are observed to be filled with colder blobs. In the pressure this creates some small-scale structure, while on large-scales the structure is seen mainly on the entropy map. There is a parcel of low entropy gas at the center of the southern part of the cluster.

On the largest scale, there is an elongation towards the north, practically in all the maps in the direction where we also see the second largest galaxy concentration in the optical 


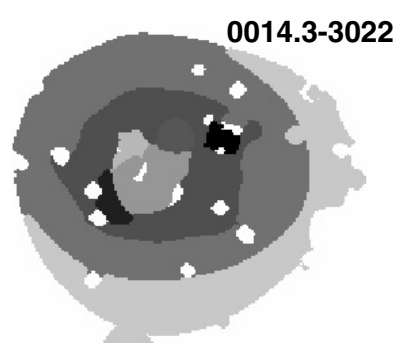

0307.0-2840
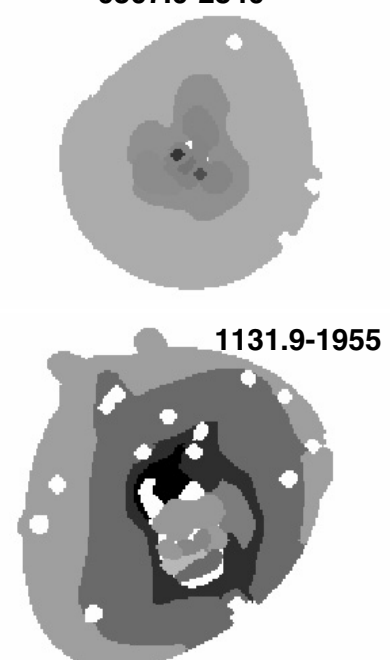

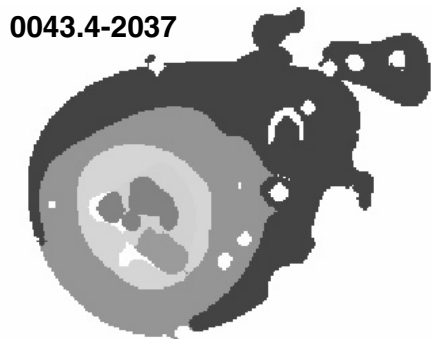

0528.9-3927

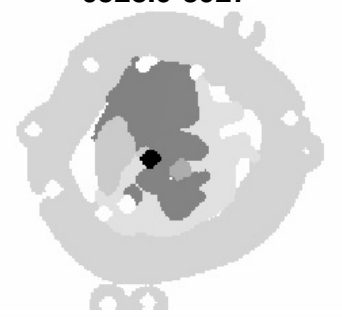

$2337.6+0016$

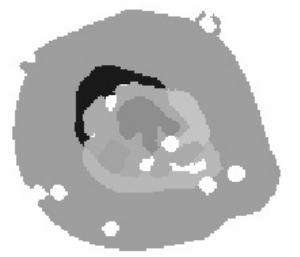

$0232.2-4420$

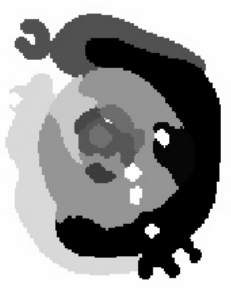

0532.9-3701

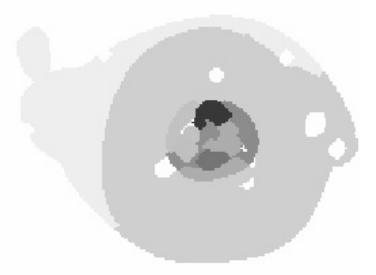

0658-5557

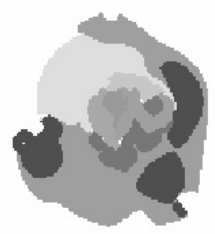

Fig. 15. Maps of the ratio between the observed pressure and the average trends measured in the DXL sample. The different shades of grey stand for a different value of the ratio, light - 0.5 (e.g. tail of RXCJ 0532.9-3701), grey - 1.1 (a dominant color of RXCJ 0532.9-3701), dark grey - 1.4 (a tail of RXCJ 0043.4-2037), black - >2.

(Böhringer et al. 2001b). This is probably due to the response of the gas pressure to the joint potential well of the two clusters that make up A1300. There is a central E-W ridge of high temperature, which may reflect the compression of the central region due to the approximately $\mathrm{N}-\mathrm{S}$ merge of the cluster.

The spectroscopic analysis is reported in Table 11 and Fig. 11. The cluster has a complex temperature structure in the core, which we assign to the distorted cool core, which is capable of partially preserving both low entropy and high pressure. Also the temperature decreases strongly towards cluster outskirts. The large scatter in the entropy profile is most probably due to the contribution of the substructure, associated with the second optical component. The outmost bin has an entropy strongly deviating from the scaling. The analysis confirms the statistical significance of the low entropy blobs in the south and reveals a pressure enhancement in the north. Combining these two features, a plausible interpretation is that the subcluster has lost its gas on approaching the cluster from the south and is currently located north of the main cluster.

\section{8. $R X C J 2337.6+0016$}

The image shows an East-West elongated core on the small scale, while on the large scale it appears to be symmetric. The pressure map has two maxima and two elongations towards the south-west and south-east. There are some ring-like structures in the temperature map. The entropy state of the cluster ICM appears to correspond to the late stage of a core disruption with filaments of the low-entropy gas spread over a large volume. The position of the entropy minimum is offset from the peak in the pressure.

The cluster has a clover leaf structure on the entropy map like RXCJ0532.9-3701. Two pressure maxima could indicate the core rebounce. Symmetry on the pressure map is regained at 1.5 arcminute radius.

The spectroscopic analysis is reported in Table 12 and Fig. 12. The scenario of the disrupted core (region 4) is supported by the spectral analysis (see also Fig. 14). The pressure enhancement (region 5) is marginal.

\subsection{RXCJ0658-5557 (the "bullet" cluster)}

Famous for its Chandra image (Markevitch et al. 2002), the bullet cluster has some distinct features, which also allow us to understand the observation of other clusters. With a Mach number of 3, deduced from the shape of the bullet itself (angle of the Mach cone), the subcluster produces an entropy enhancement in front of it. There are two other large entropy peaks behind and to the south of the bullet. Apart from the small-scale structure in the center, there appears to be a lack of features on the pressure map, which we attribute to propagation of the shock out to large radii, thus strongly reducing the contrast. Therefore, the bullet indicates a situation of a strong merger that is just completed in the center and now moves to the 
Table 7. Properties of the main regions of RXCJ0232.2-4420.

\begin{tabular}{ccccccccc}
\hline \hline$N$ & $\begin{array}{c}k T \\
\mathrm{keV}\end{array}$ & $\begin{array}{c}\rho_{e} \\
10^{-4} \mathrm{~cm}^{-3}\end{array}$ & $\begin{array}{c}S \\
\mathrm{keV} \mathrm{cm}^{2}\end{array}$ & $\begin{array}{c}P, 10^{-12} \\
\mathrm{egs} \mathrm{cm}^{-3}\end{array}$ & $\begin{array}{c}M_{\text {gas }} \\
10^{12} M_{\odot}\end{array}$ & $\begin{array}{c}r_{\min } \\
\mathrm{Mpc}\end{array}$ & $\begin{array}{c}r_{\max } \\
\mathrm{Mpc}\end{array}$ & Remarks \\
\hline 1 & $8.9 \pm 2.6$ & $34.8 \pm 4.3$ & $388 \pm 119$ & $49.8 \pm 15.9$ & $1.1 \pm 0.1$ & 0.21 & 0.36 & \\
2 & $8.0 \pm 1.2$ & $42.1 \pm 3.0$ & $305 \pm 50$ & $53.7 \pm 9.2$ & $1.3 \pm 0.1$ & 0.16 & 0.29 & \\
3 & $5.1 \pm 0.2$ & $169.0 \pm 4.3$ & $77 \pm 4$ & $136.9 \pm 7.3$ & $1.7 \pm 0.0$ & 0.00 & 0.10 & cool core \\
4 & $7.6 \pm 0.5$ & $54.3 \pm 1.3$ & $245 \pm 17$ & $65.9 \pm 4.7$ & $5.7 \pm 0.1$ & 0.08 & 0.32 & \\
5 & $8.5 \pm 2.0$ & $51.3 \pm 4.8$ & $287 \pm 68$ & $70.1 \pm 17.4$ & $0.8 \pm 0.1$ & 0.15 & 0.24 & \\
6 & $6.3 \pm 1.0$ & $40.1 \pm 3.2$ & $248 \pm 43$ & $40.3 \pm 7.4$ & $2.0 \pm 0.2$ & 0.14 & 0.36 & \\
7 & $7.9 \pm 2.6$ & $12.6 \pm 1.7$ & $677 \pm 235$ & $15.9 \pm 5.7$ & $3.6 \pm 0.5$ & 0.43 & 0.79 & \\
8 & $6.5 \pm 0.4$ & $12.7 \pm 0.3$ & $557 \pm 39$ & $13.3 \pm 1.0$ & $24.5 \pm 0.6$ & 0.24 & 0.76 & \\
9 & $5.0 \pm 0.7$ & $19.8 \pm 1.5$ & $315 \pm 46$ & $15.7 \pm 2.4$ & $4.2 \pm 0.3$ & 0.19 & 0.53 & entropy tail \\
10 & $6.9 \pm 2.7$ & $39.8 \pm 11$. & $275 \pm 118$ & $44.0 \pm 21.3$ & $0.8 \pm 0.2$ & 0.18 & 0.35 & \\
11 & $8.9 \pm 2.8$ & $4.4 \pm 0.3$ & $1532 \pm 494$ & $6.4 \pm 2.1$ & $17.7 \pm 1.3$ & 0.57 & 1.27 & forward shock \\
12 & $3.1 \pm 0.5$ & $5.6 \pm 0.7$ & $448 \pm 83$ & $2.8 \pm 0.6$ & $10.0 \pm 1.3$ & 0.48 & 1.11 & outskirts \\
13 & $8.3 \pm 4.3$ & $4.5 \pm 0.9$ & $1405 \pm 749$ & $6.0 \pm 3.3$ & $5.7 \pm 1.2$ & 0.52 & 1.12 & \\
\hline
\end{tabular}

Table 8. Properties of the main regions of RXCJ0307.0-2840.

\begin{tabular}{ccccccccc}
\hline \hline$N$ & $\begin{array}{c}k T \\
\mathrm{keV}\end{array}$ & $\begin{array}{c}\rho_{e} \\
10^{-4} \mathrm{~cm}^{-3}\end{array}$ & $\begin{array}{c}S \\
\mathrm{keV} \mathrm{cm}^{2}\end{array}$ & $\begin{array}{c}P, 10^{-12} \\
\mathrm{ergs} \mathrm{cm}^{-3}\end{array}$ & $\begin{array}{c}M_{\text {gas }} \\
10^{12} M_{\odot}\end{array}$ & $\begin{array}{c}r_{\min } \\
\mathrm{Mpc}\end{array}$ & $\begin{array}{c}r_{\max } \\
\mathrm{Mpc}\end{array}$ & Remarks \\
\hline 1 & $6.3 \pm 1.2$ & $85.9 \pm 12.2$ & $151 \pm 31$ & $87.4 \pm 20.3$ & $0.3 \pm 0.0$ & 0.07 & 0.14 & \\
2 & $6.1 \pm 1.6$ & $72.2 \pm 12.9$ & $163 \pm 47$ & $70.4 \pm 22.3$ & $0.2 \pm 0.0$ & 0.09 & 0.16 & \\
3 & $5.5 \pm 0.3$ & $64.7 \pm 1.6$ & $157 \pm 10$ & $56.6 \pm 3.8$ & $4.1 \pm 0.1$ & 0.02 & 0.22 & \\
4 & $4.3 \pm 0.3$ & $157.7 \pm 8.6$ & $68 \pm 5$ & $108.5 \pm 9.2$ & $0.6 \pm 0.0$ & 0.00 & 0.09 & cool core \\
5 & $6.8 \pm 0.7$ & $21.3 \pm 1.2$ & $408 \pm 46$ & $23.1 \pm 2.8$ & $2.7 \pm 0.2$ & 0.17 & 0.43 & \\
6 & $5.7 \pm 0.8$ & $25.2 \pm 1.9$ & $307 \pm 45$ & $22.9 \pm 3.6$ & $2.1 \pm 0.2$ & 0.19 & 0.43 & \\
7 & $6.4 \pm 1.3$ & $28.6 \pm 2.2$ & $318 \pm 66$ & $29.5 \pm 6.4$ & $1.4 \pm 0.1$ & 0.17 & 0.35 & \\
8 & $5.9 \pm 0.5$ & $14.3 \pm 0.4$ & $465 \pm 37$ & $13.6 \pm 1.1$ & $14.1 \pm 0.4$ & 0.16 & 0.68 & \\
9 & $4.7 \pm 0.5$ & $4.4 \pm 0.2$ & $808 \pm 83$ & $3.3 \pm 0.3$ & $44.5 \pm 1.8$ & 0.31 & 1.17 & \\
\hline
\end{tabular}

Table 9. Properties of the main regions of RXCJ0528.9-3927. Region 1 turned out to be a QSO, so is omitted.

\begin{tabular}{ccccccccc}
\hline \hline$N$ & $\begin{array}{c}k T \\
\mathrm{keV}\end{array}$ & $\begin{array}{c}\rho_{e} \\
10^{-4} \mathrm{~cm}^{-3}\end{array}$ & $\begin{array}{c}S \\
\mathrm{keV} \mathrm{cm}^{2}\end{array}$ & $\begin{array}{c}P, 10^{-12} \\
\mathrm{ergs} \mathrm{cm}^{-3}\end{array}$ & $\begin{array}{c}M_{\text {gas }} \\
10^{12} M_{\odot}\end{array}$ & $\begin{array}{c}r_{\min } \\
\mathrm{Mpc}\end{array}$ & $\begin{array}{c}r_{\max } \\
\mathrm{Mpc}\end{array}$ & Remarks \\
\hline 2 & $4.7 \pm 0.5$ & $93.8 \pm 6.0$ & $106 \pm 12$ & $70.6 \pm 9.0$ & $2.0 \pm 0.1$ & 0.00 & 0.17 & core region \\
3 & $6.2 \pm 0.7$ & $41.2 \pm 2.5$ & $242 \pm 30$ & $41.1 \pm 5.4$ & $6.1 \pm 0.4$ & 0.04 & 0.32 & \\
4 & $6.5 \pm 1.4$ & $34.3 \pm 5.0$ & $288 \pm 66$ & $35.9 \pm 9.1$ & $2.1 \pm 0.3$ & 0.15 & 0.34 & \\
5 & $5.4 \pm 1.7$ & $6.0 \pm 0.4$ & $764 \pm 244$ & $5.2 \pm 1.7$ & $9.1 \pm 0.7$ & 0.46 & 1.31 & \\
6 & $4.6 \pm 0.7$ & $13.8 \pm 1.4$ & $370 \pm 58$ & $10.1 \pm 1.8$ & $10.2 \pm 1.0$ & 0.22 & 0.72 & \\
7 & $5.8 \pm 0.7$ & $6.8 \pm 0.3$ & $755 \pm 89$ & $6.3 \pm 0.8$ & $54.6 \pm 2.1$ & 0.16 & 1.27 & \\
\hline
\end{tabular}

Table 10. Properties of the main regions of RXCJ0532.9-3701.

\begin{tabular}{ccccccccc}
\hline \hline$N$ & $\begin{array}{c}k T \\
\mathrm{keV}\end{array}$ & $\begin{array}{c}\rho_{e} \\
10^{-4} \mathrm{~cm}^{-3}\end{array}$ & $\begin{array}{c}S \\
\mathrm{keV} \mathrm{cm}^{2}\end{array}$ & $\begin{array}{c}P, 10^{-12} \\
\mathrm{ergs} \mathrm{cm} \mathrm{cm}^{-3}\end{array}$ & $\begin{array}{c}M_{\text {gas }} \\
10^{12} M_{\odot}\end{array}$ & $\begin{array}{c}r_{\min } \\
\mathrm{Mpc}\end{array}$ & $\begin{array}{c}r_{\max } \\
\mathrm{Mpc}\end{array}$ & Remarks \\
\hline 1 & $6.0 \pm 1.1$ & $36.0 \pm 3.9$ & $256 \pm 52$ & $34.6 \pm 7.6$ & $1.1 \pm 0.1$ & 0.13 & 0.31 & \\
2 & $11.6 \pm 2.9$ & $39.7 \pm 3.1$ & $461 \pm 118$ & $73.6 \pm 19.3$ & $2.0 \pm 0.2$ & 0.12 & 0.31 & \\
3 & $8.6 \pm 3.2$ & $48.9 \pm 5.3$ & $300 \pm 112$ & $67.7 \pm 25.8$ & $1.4 \pm 0.1$ & 0.09 & 0.24 & \\
4 & $6.5 \pm 0.9$ & $31.1 \pm 1.8$ & $307 \pm 43$ & $32.6 \pm 4.8$ & $2.4 \pm 0.1$ & 0.10 & 0.36 & \\
5 & $6.7 \pm 0.5$ & $43.0 \pm 1.2$ & $253 \pm 18$ & $46.1 \pm 3.4$ & $5.9 \pm 0.2$ & 0.00 & 0.36 & core \\
6 & $6.9 \pm 0.7$ & $47.6 \pm 2.0$ & $243 \pm 27$ & $52.5 \pm 6.1$ & $2.9 \pm 0.1$ & 0.02 & 0.37 & \\
7 & $7.3 \pm 1.7$ & $30.4 \pm 3.0$ & $349 \pm 84$ & $35.6 \pm 9.0$ & $2.3 \pm 0.2$ & 0.20 & 0.37 & \\
8 & $1.4 \pm 0.3$ & $3.3 \pm 0.6$ & $286 \pm 65$ & $0.7 \pm 0.2$ & $17.0 \pm 3.2$ & 0.84 & 1.71 & filament \\
9 & $4.5 \pm 0.4$ & $5.6 \pm 0.2$ & $665 \pm 55$ & $4.1 \pm 0.4$ & $57.0 \pm 1.7$ & 0.30 & 1.15 & \\
\hline
\end{tabular}


Table 11. Properties of the main regions of RXCJ1131.9-1955.

\begin{tabular}{ccccccccc}
\hline \hline$N$ & $\begin{array}{c}k T \\
\mathrm{keV}\end{array}$ & $\begin{array}{c}\rho_{e} \\
10^{-4} \mathrm{~cm}^{-3}\end{array}$ & $\begin{array}{c}S \\
\mathrm{keV} \mathrm{cm}^{2}\end{array}$ & $\begin{array}{c}P, 10^{-12} \\
\mathrm{ergs} \mathrm{cm}^{-3}\end{array}$ & $\begin{array}{c}M_{\text {gas }} \\
10^{12} M_{\odot}\end{array}$ & $\begin{array}{c}r_{\min } \\
\mathrm{Mpc}\end{array}$ & $\begin{array}{c}r_{\max } \\
\mathrm{Mpc}\end{array}$ & Remarks \\
\hline 1 & $8.5 \pm 0.6$ & $32.4 \pm 0.8$ & $387 \pm 28$ & $43.9 \pm 3.2$ & $9.4 \pm 0.2$ & 0.03 & 0.39 & \\
2 & $7.4 \pm 1.3$ & $29.1 \pm 1.8$ & $363 \pm 64$ & $34.5 \pm 6.3$ & $2.3 \pm 0.1$ & 0.21 & 0.41 & \\
3 & $5.1 \pm 0.5$ & $33.2 \pm 2.9$ & $231 \pm 27$ & $27.3 \pm 3.6$ & $1.6 \pm 0.1$ & 0.15 & 0.38 & \\
4 & $5.5 \pm 0.8$ & $45.2 \pm 4.0$ & $201 \pm 33$ & $39.7 \pm 7.0$ & $1.4 \pm 0.1$ & 0.14 & 0.31 & \\
5 & $6.0 \pm 0.4$ & $79.1 \pm 2.5$ & $151 \pm 11$ & $76.0 \pm 5.9$ & $2.3 \pm 0.1$ & 0.02 & 0.19 & \\
6 & $2.1 \pm 0.4$ & $2.7 \pm 0.5$ & $503 \pm 107$ & $0.9 \pm 0.2$ & $34.7 \pm 5.9$ & 0.89 & 1.95 & \\
7 & $4.5 \pm 0.5$ & $4.3 \pm 0.2$ & $797 \pm 89$ & $3.1 \pm 0.4$ & $58.5 \pm 2.6$ & 0.39 & 1.59 & \\
8 & $6.9 \pm 0.8$ & $10.1 \pm 0.3$ & $680 \pm 81$ & $11.2 \pm 1.3$ & $20.6 \pm 0.6$ & 0.29 & 0.98 & \\
9 & $10.6 \pm 2.0$ & $13.3 \pm 0.6$ & $875 \pm 169$ & $22.7 \pm 4.4$ & $4.7 \pm 0.2$ & 0.35 & 0.79 & \\
10 & $8.0 \pm 1.4$ & $15.5 \pm 1.3$ & $599 \pm 111$ & $19.9 \pm 3.9$ & $3.5 \pm 0.3$ & 0.25 & 0.55 & \\
\hline
\end{tabular}

Table 12. Properties of the main regions of RXCJ2337.6+0016.

\begin{tabular}{ccccccccc}
\hline \hline$N$ & $\begin{array}{c}k T \\
\mathrm{keV}\end{array}$ & $\begin{array}{c}\rho_{e} \\
10^{-4} \mathrm{~cm}^{-3}\end{array}$ & $\begin{array}{c}S \\
\mathrm{keV} \mathrm{cm}^{2}\end{array}$ & $\begin{array}{c}P, 10^{-12} \\
\mathrm{ergs} \mathrm{cm}^{-3}\end{array}$ & $\begin{array}{c}M_{\text {gas }} \\
10^{12} M_{\odot}\end{array}$ & $\begin{array}{c}r_{\min } \\
\mathrm{Mpc}\end{array}$ & $\begin{array}{c}r_{\max } \\
\mathrm{Mpc}\end{array}$ & Remarks \\
\hline 1 & $7.6 \pm 2.0$ & $21.3 \pm 3.4$ & $460 \pm 133$ & $25.9 \pm 8.1$ & $1.5 \pm 0.2$ & 0.18 & 0.43 & \\
2 & $9.2 \pm 1.0$ & $37.3 \pm 1.1$ & $381 \pm 42$ & $54.7 \pm 6.2$ & $6.0 \pm 0.2$ & 0.00 & 0.34 & \\
3 & $7.6 \pm 0.6$ & $21.3 \pm 0.5$ & $456 \pm 38$ & $25.9 \pm 2.2$ & $13.0 \pm 0.3$ & 0.05 & 0.54 & \\
4 & $4.7 \pm 0.7$ & $20.7 \pm 1.9$ & $292 \pm 47$ & $15.7 \pm 2.7$ & $2.9 \pm 0.3$ & 0.24 & 0.50 & disrupted core \\
5 & $8.8 \pm 3.2$ & $12.7 \pm 1.5$ & $751 \pm 281$ & $17.8 \pm 6.9$ & $3.5 \pm 0.4$ & 0.41 & 0.69 & secondary pressure peak? \\
6 & $7.2 \pm 2.0$ & $10.9 \pm 1.4$ & $681 \pm 202$ & $12.5 \pm 3.9$ & $4.0 \pm 0.5$ & 0.26 & 0.64 & \\
7 & $5.9 \pm 0.6$ & $4.5 \pm 0.2$ & $997 \pm 113$ & $4.3 \pm 0.5$ & $41.2 \pm 1.8$ & 0.41 & 1.17 & \\
\hline
\end{tabular}

Table 13. Properties of the main regions of RXCJ0658-5557.

\begin{tabular}{ccccccccc}
\hline \hline$N$ & $\begin{array}{c}k T \\
\mathrm{keV}\end{array}$ & $\begin{array}{c}\rho_{e} \\
10^{-4} \mathrm{~cm}^{-3}\end{array}$ & $\begin{array}{c}S \\
\mathrm{keV} \mathrm{cm}^{2}\end{array}$ & $\begin{array}{c}P, 10^{-12} \\
\mathrm{ergs} \mathrm{cm}^{-3}\end{array}$ & $\begin{array}{c}M_{\text {gas }} \\
10^{12} M_{\odot}\end{array}$ & $\begin{array}{c}r_{\text {min }} \\
\mathrm{Mpc}\end{array}$ & $\begin{array}{c}r_{\text {max }} \\
\mathrm{Mpc}\end{array}$ & Remarks \\
\hline 1 & $12.1 \pm 1.0$ & $85.2 \pm 1.9$ & $290 \pm 26$ & $165.3 \pm 14.8$ & $2.7 \pm 0.1$ & 0.00 & 0.22 & P core-1 \\
2 & $14.0 \pm 1.8$ & $10.8 \pm 0.3$ & $1332 \pm 169$ & $24.3 \pm 3.1$ & $18.6 \pm 0.5$ & 0.45 & 1.12 & shock \\
3 & $10.4 \pm 0.6$ & $21.3 \pm 0.3$ & $626 \pm 35$ & $35.3 \pm 2.0$ & $20.6 \pm 0.3$ & 0.14 & 0.63 & tail \\
4 & $8.7 \pm 1.0$ & $4.0 \pm 0.1$ & $1593 \pm 190$ & $5.6 \pm 0.7$ & $59.6 \pm 1.5$ & 0.61 & 1.79 & main-2 \\
5 & $12.2 \pm 0.9$ & $34.2 \pm 0.6$ & $540 \pm 42$ & $67.1 \pm 5.3$ & $8.8 \pm 0.2$ & 0.24 & 0.58 & P core-3 \\
6 & $9.4 \pm 0.7$ & $43.1 \pm 0.9$ & $354 \pm 27$ & $64.6 \pm 5.0$ & $4.4 \pm 0.1$ & 0.11 & 0.45 & bullet tail N \\
7 & $9.9 \pm 0.6$ & $64.3 \pm 1.1$ & $286 \pm 17$ & $101.8 \pm 6.2$ & $4.0 \pm 0.1$ & 0.11 & 0.32 & bullet tail W \\
8 & $11.4 \pm 1.0$ & $51.0 \pm 1.0$ & $383 \pm 33$ & $92.9 \pm 8.0$ & $5.1 \pm 0.1$ & 0.07 & 0.39 & P core-2 \\
9 & $10.0 \pm 0.5$ & $12.2 \pm 0.2$ & $871 \pm 48$ & $19.5 \pm 1.1$ & $42.0 \pm 0.6$ & 0.30 & 1.13 & main-1 \\
10 & $8.0 \pm 0.6$ & $64.7 \pm 1.9$ & $230 \pm 17$ & $82.5 \pm 6.5$ & $1.6 \pm 0.0$ & 0.27 & 0.43 & bullet peak \\
11 & $7.7 \pm 0.5$ & $45.8 \pm 0.9$ & $281 \pm 17$ & $56.9 \pm 3.5$ & $4.1 \pm 0.1$ & 0.25 & 0.52 & bullet front \\
\hline
\end{tabular}

outskirts. The entropy structure of the core of the main cluster appears disrupted, yet the minimum is retained, while becoming shallow. In the temperature map we see clear signatures of turbulence, as indicated by the stochastic fluctuations, which in other clusters correspond to a late stage of merging. This once again demonstrates that the time scales for the relaxation are very different for the cluster center and outskirts. On the largest scale the pressure, as well as the image, appears to be quite smooth.

A weak lensing mass reconstruction of Clowe et al. (2003) shows that the cluster exhibits three dark matter peaks, with only the weakest of them corresponding to an X-ray peak; yet all of them are preceded by a shocked zone, as seen on the entropy map. The main pressure peak is located approximately at the position of the center of the mass distribution from the weak lensing reconstruction. This center is adopted for the volume calculations and reported in Table 1. The entropy dip of the bullet is offset from the potential minimum and there is no entropy dip associated with the potential minimum of the main cluster. There are, however, entropy fluctuations in the pressure core, possibly associated with debris of the entropy core of the main cluster.

The spectroscopic analysis is reported in Table 13 and Fig. 13. It reveals temperature fluctuations by a factor of 1.5 . 
The temperature of the bullet is only slightly lower than the bulk of the cluster; however, it exhibits a distinctly low entropy, which also allows us to trace the tail of the bullet. The zone assigned to the bullet can be seen as negative deviation in the entropy profile in Fig. 13. The bullet pressure peak is confirmed; it amounts to $20 \%$ and is located behind the zone of lowest entropy in the bullet.

By combining all the high-entropy zones associated with the shock heating, we have achieved $99 \%$ significance in the temperature variation, from 10 . to $14 \pm 2 \mathrm{keV}$. This corresponds to a Mach number of $1.4 \pm 0.2$. This estimate is lower than the shock parameters deduced from the image showing the Mach cone. A higher Mach number would be obtained from the entropy enhancement: $2.6 \pm 0.2$. It is plausible that the extraction region captures both shock and postshock gas. The latter has lower pressure, but records its state in the entropy. As noted above, the observed shock is located in front of the outward moving dark matter potential. Since the potentials no longer carry any gas, they do not cause this shock, but just travel at the same speed. This implies that we observe the initial forward shock propagating through the cluster.

The entropy ratio also shows that the eastern part of the cluster has lower entropy, due to the stripping of the bulk of the bullet cluster.

\section{Discussion and summary}

Analysis of the two-dimensional structure in the REFLEX clusters, as seen in the images and spectral hardness ratio maps, reveals statistically significant substructure, probably originating from different stages of cluster merger. We are able to see the substructure even at very late merger stages, where for example the X-ray image appears to be quite symmetric. We identify the entropy to be most sensitive to both late stage mergers with the associated slow buoyancy action of relaxation of the cluster and to strong shocks, which change the entropy. Two mergers with large Mach numbers are found.

A statistical analysis of the substructure in the pressure and entropy maps reveals significant fluctuations around the mean profile. Typically, pressure fluctuations are found at the $30 \%$ level, while the entropy fluctuations are at the $20 \%$ level. Apparently, smoother appearance of the pressure maps should be attributed to the larger dynamical range of the map, typically covering two orders of magnitude. A comparison of our sample with a similar analysis of hydro-dynamical simulations by Finoguenov et al. (2005) reveals a similar distribution of clusters vs the level of the substructure in both entropy and pressure.

A number of clusters exhibit a presence of low entropy gas in the outskirts, deviating by at least an order of magnitude from the prescription of gravitational heating. Surprisingly enough, these regions have gas pressures similar to that of the cluster at a similar distance from the center. This argues in favor of these regions being embedded in the cluster gas and maintaining the pressure equilibrium, therefore revealing a medium survived from the accretion shock heating. Existence of this effect has been suggested by the simulations (e.g. Motl et al. 2004), but has so far only been reported for A85 (Durret et al. 2005).

Incomplete (in the sense of ongoing) shock propagation in clusters soon after the major merging event could also be a cause of the low entropies seen at the outskirts. In fact, the clusters at the advanced stage of interaction have systematically higher entropy at $r_{500}$ compared to the average trend.

Acknowledgements. This paper is based on observations obtained with XMM-Newton, an ESA science mission, with instruments and contributions directly funded by ESA Member States and the USA (NASA). The XMM-Newton project is supported by the Bundesministerium für Bildung und Forschung/Deutsches Zentrum für Luft- und Raumfahrt (BMFT/DLR), the Max-Planck Society and the Heidenhain-Stiftung, and also by PPARC, CEA, CNES, and ASI. The authors thank the referee, Florence Durret, for the constructive comments and her appreciation of their efforts. AF thanks Alastair Sanderson and Joe Mohr for useful discussions. Partial support from NASA grant NNG04GF68G to UMBC is acknowledged. A.F. acknowledges support from BMBF/DLR under grant 50 OR 0207 and MPG.

\section{References}

Belsole, E., Sauvageot, J.-L., Pratt, G. W., \& Bourdin, H. 2005, A\&A, 430, 385

Böhringer, H., Schuecker, P., Guzzo, L., et al. 2001a, A\&A, 369, 826

Böhringer, H., Schuecker, P., Lynam, P., et al. 2001b, The Messenger, 106,24

Briel, U. G., Finoguenov, A., \& Henry, J. P. 2004, A\&A, 426, 1

Churazov, E., Gilfanov, M., Forman, W., \& Jones, C. 1999, ApJ, 520, 105

Clowe, D., Gonzalez, A., \& Markevitch, M. 2004, ApJ, 604, 596

De Luca, A., \& Molendi, S. 2004, A\&A, 419, 837

Durret, F., Lima Neto, G. B., \& Forman, W. 2005, A\&A, 432, 809

Finoguenov, A., Reiprich, T. H., \& Böhringer, H. 2001, A\&A, 368, 749

Finoguenov, A., Mohr, J. J., Bialek, J. J., Evrard, A., \& Sanderson, A. J. R. 2005, ApJ, submitted

Jones, C., \& Forman, W. 1984, ApJ, 276, 38

Henry, J. P., Finoguenov, A., \& Briel, U. G. 2004, ApJ, 615, 181

Kempner, J. C., \& David, L. P. 2004, MNRAS, 349, 385

Markevitch, M., Gonzalez, A., David, L., et al. 2002, ApJ, 567, 27

Mahdavi, A., Finoguenov, A., Böhringer, H., Geller, M. J., \& Henry, J. P. 2005, ApJ, 622, 187

Motl, P. M., Burns, J. O., Loken, C., Norman, M. L., \& Bryan, G. 2004, ApJ, 606, 635

Ponman, T. J., Sanderson, A. J. R., \& Finoguenov, A. 2003, MNRAS, 343,331

Randall, S. W., Sarazin, C. L., \& Ricker, P. M. 2002, ApJ, 577, 579

Reiprich, T. H., Sarazin, C. L., Kempner, J. C., \& Tittley, E. 2004, ApJ, 608, 179

Rowley, D. R., Thomas, P. A., \& Kay, S. T. 2004, MNRAS, 352, 508

Sanderson, A. J. R., Finoguenov, A., \& Mohr, J. J. 2005, ApJ, in press [arXiv: astro-ph/0412316]

Vikhlinin, A., Mc Namara, B. R., Forman, W., et al. 1998, ApJ, 502, 558

Vikhlinin, A., Markevitch, M., Murray, S. S., et al. 2005, ApJ, in press [arXiv:astro-ph/0412306]

Voit, G. M., \& Bryan, G. L. 2001, Nature, 414, 425

Voit, G. M. 2005, RvMP, 77, 207

Zhang, Y.-Y., Finoguenov, A., Böhringer, H., et al. 2004, A\&A, 413, 49

Zhang, Y.-Y., et al. 2005, A\&A, submitted 
A. Finoguenov et al.: XMM-Newton maps of DXL clusters, Online Material p 1

\section{Online Material}


A. Finoguenov et al.: XMM-Newton maps of DXL clusters, Online Material $p 2$
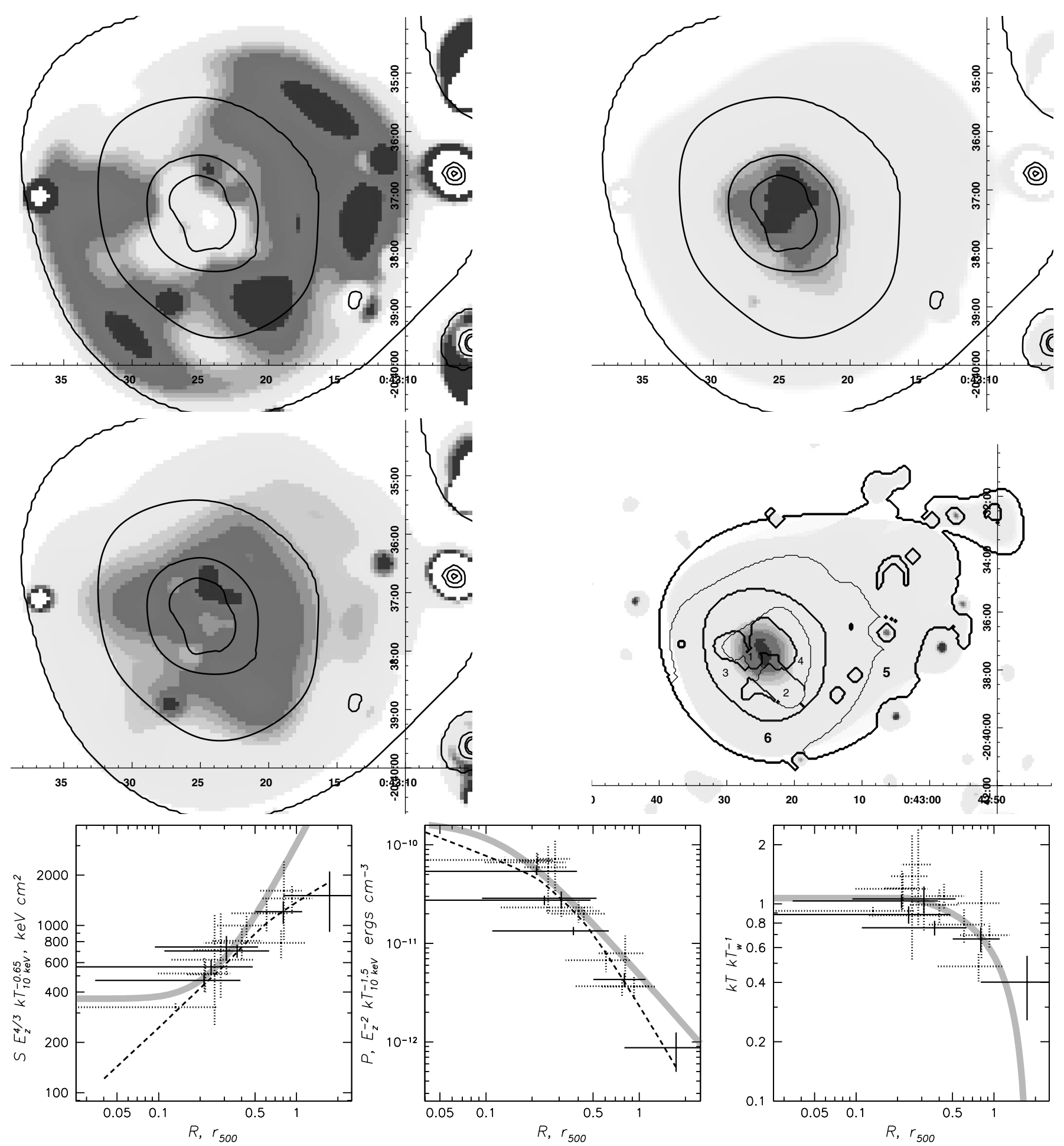

Fig. 6. RXCJ0043.4-2037. Explanations are similar to Fig. 5. The surface brightness image is overlaid with contours showing the spectral extraction regions with numbers corresponding to those in Table 6 . 
A. Finoguenov et al.: XMM-Newton maps of DXL clusters, Online Material p 3
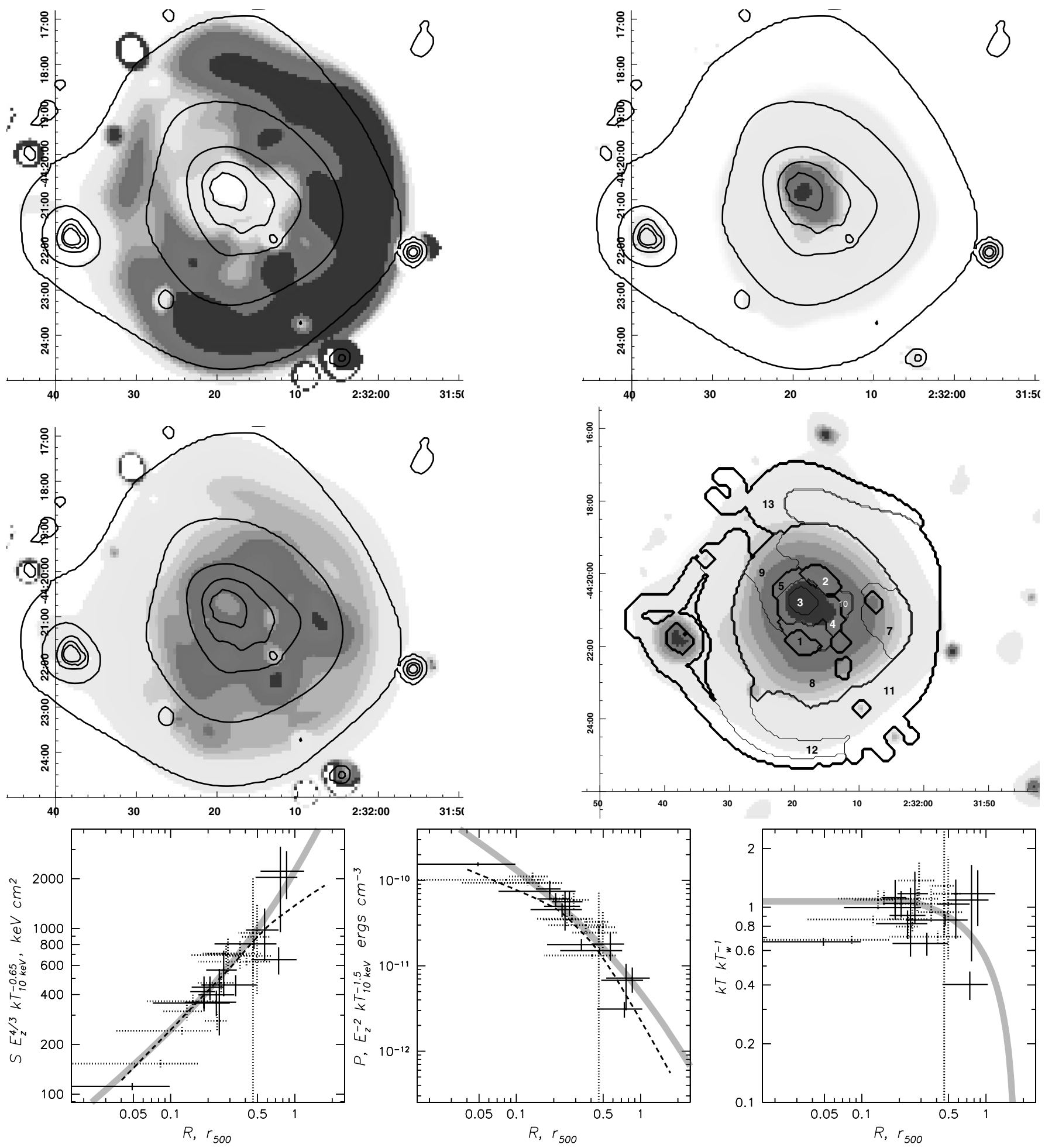

Fig. 7. RXCJ0232.2-4420. Explanations are similar to Fig. 5. The surface brightness image is overlaid with contours showing the spectral extraction regions with numbers corresponding to those in Table 7. 
A. Finoguenov et al.: XMM-Newton maps of DXL clusters, Online Material $p 4$


Fig. 8. RXCJ0307.0-2840. Explanations are similar to Fig. 5. The surface brightness image is overlaid with contours showing the spectral extraction regions with numbers corresponding to those in Table 8 . 
A. Finoguenov et al.: XMM-Newton maps of DXL clusters, Online Material p 5
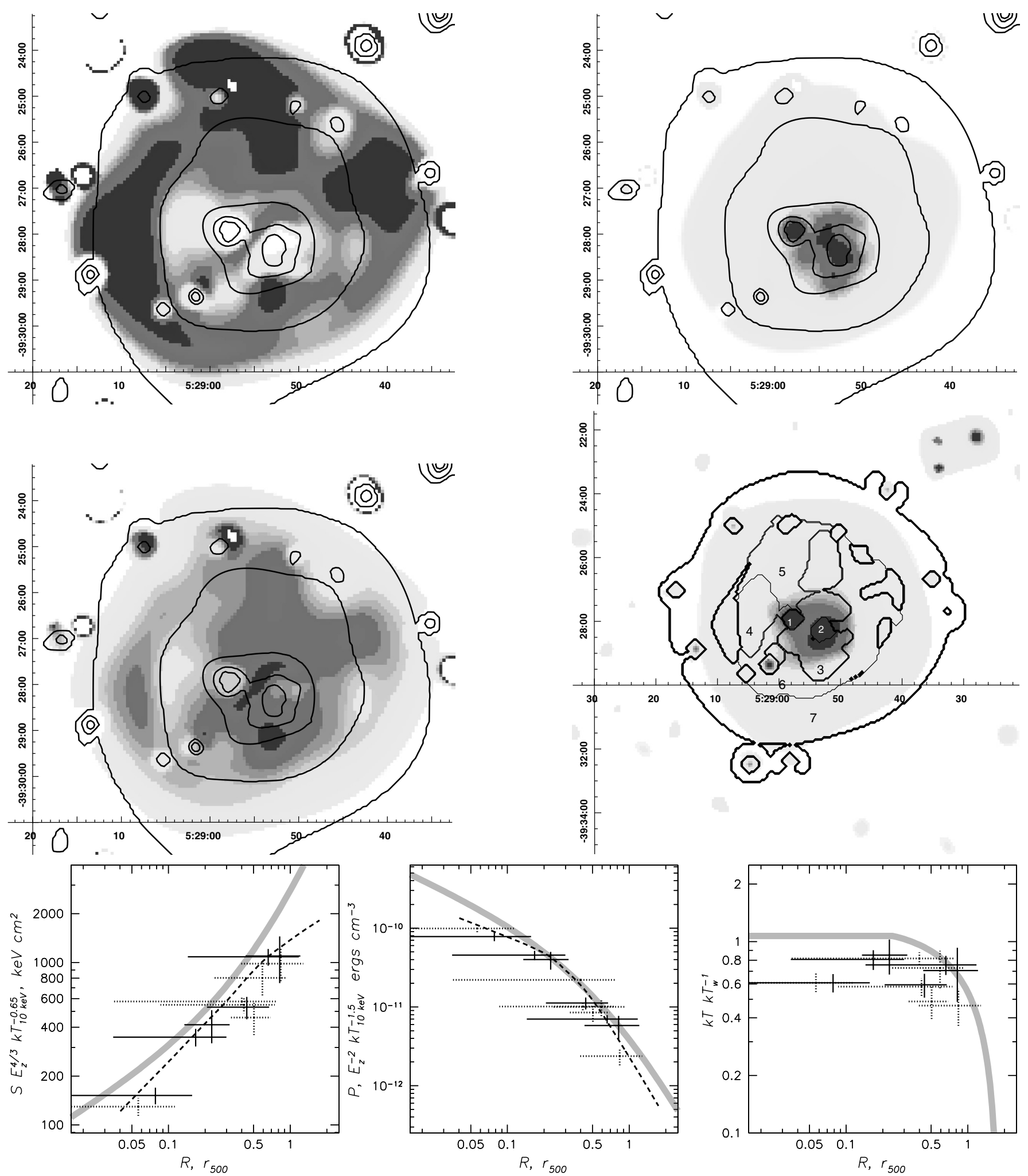

Fig. 9. RXCJ0528.9-3927. Explanations are similar to Fig. 5. The surface brightness image is overlaid with contours showing the spectral extraction regions with numbers corresponding to those in Table 9. 
A. Finoguenov et al.: XMM-Newton maps of DXL clusters, Online Material p 6
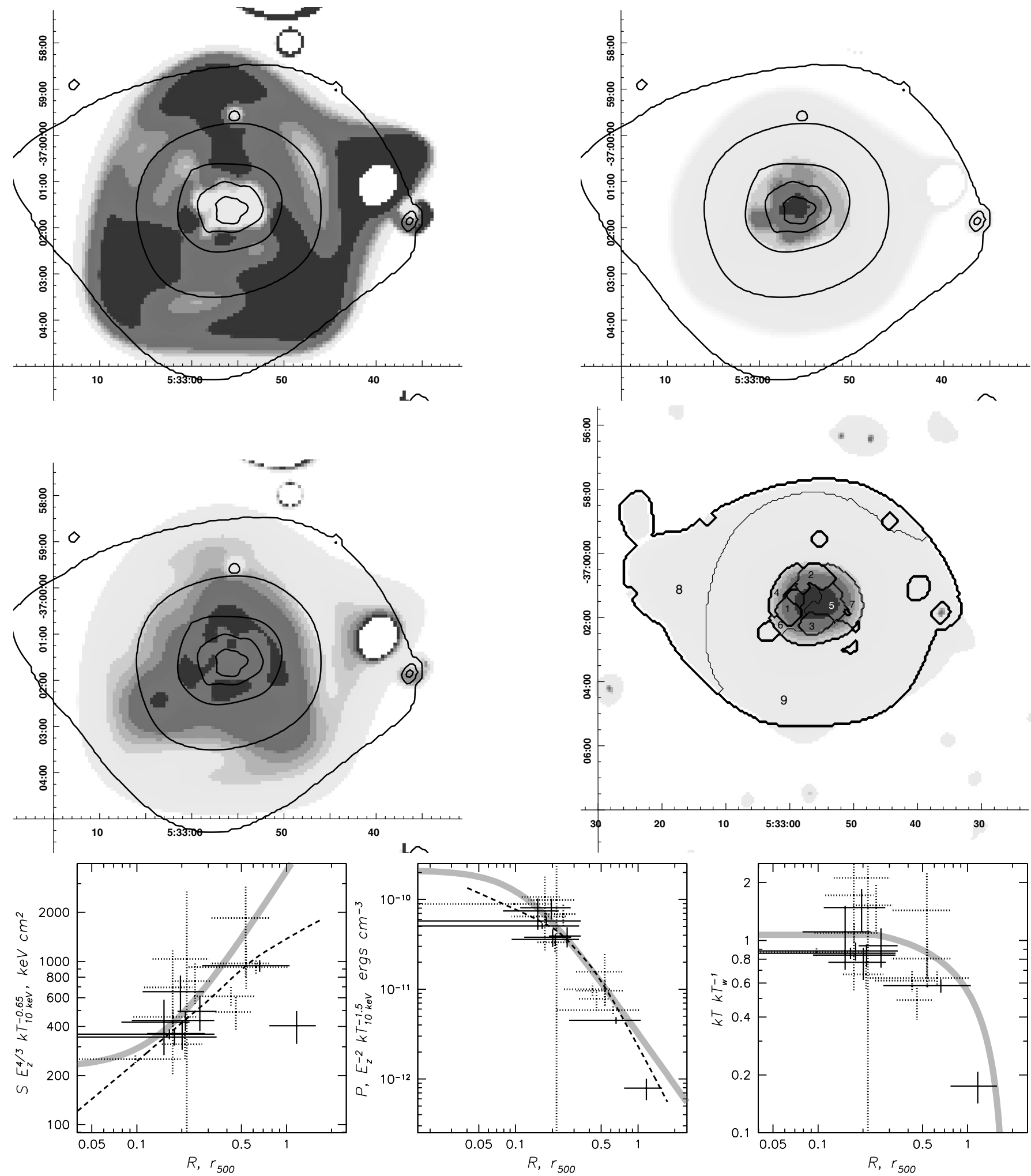

Fig. 10. RXCJ0532.9-3701. Explanations are similar to Fig. 5. The surface brightness image is overlaid with contours showing the spectral extraction regions with numbers corresponding to those in Table 10. 
A. Finoguenov et al.: XMM-Newton maps of DXL clusters, Online Material $p 7$
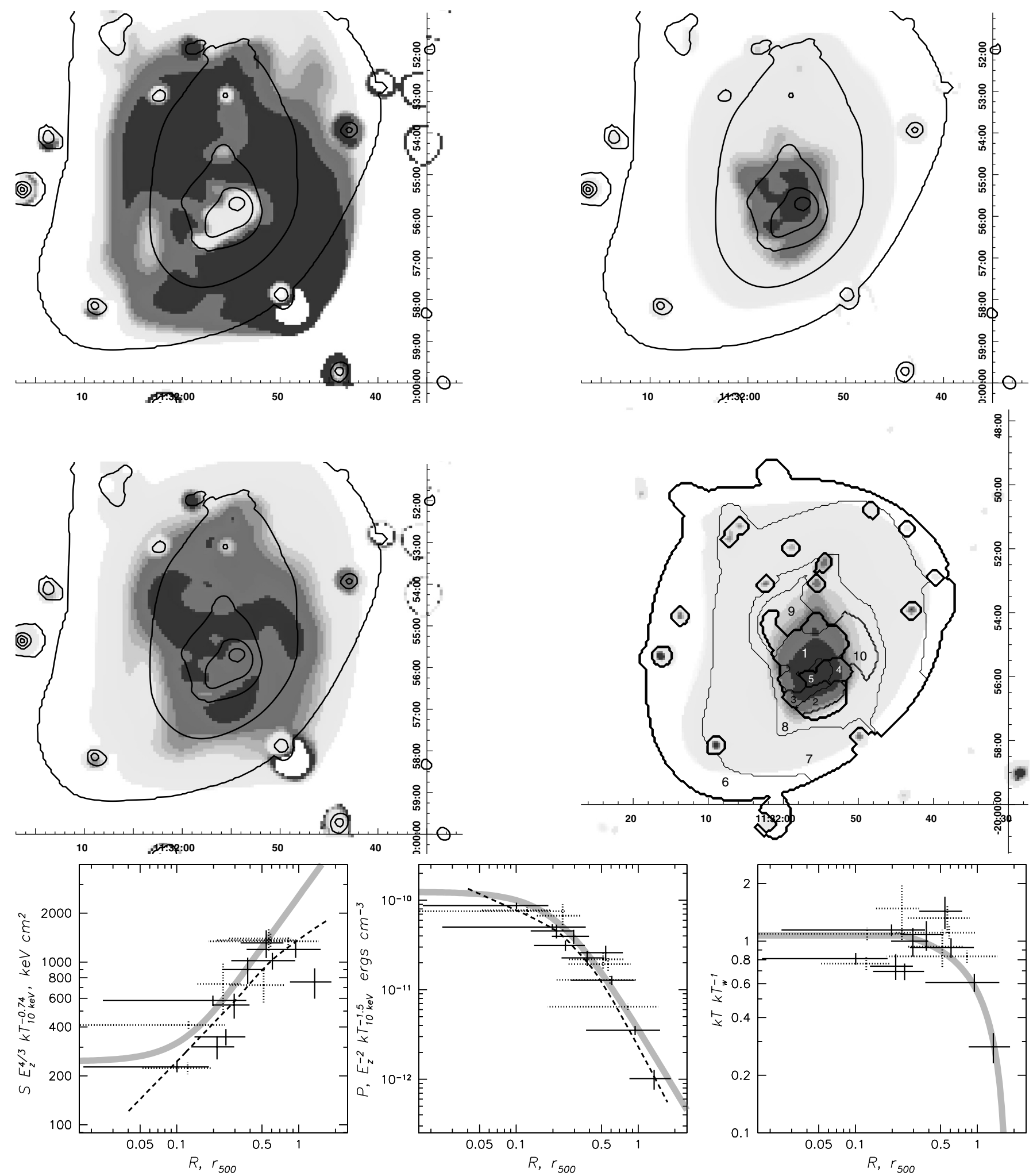

Fig. 11. RXCJ1131.9-1955. Explanations are similar to Fig. 5. The surface brightness image is overlaid with contours showing the spectral extraction regions with numbers corresponding to those in Table 11. 
A. Finoguenov et al.: XMM-Newton maps of DXL clusters, Online Material p 8
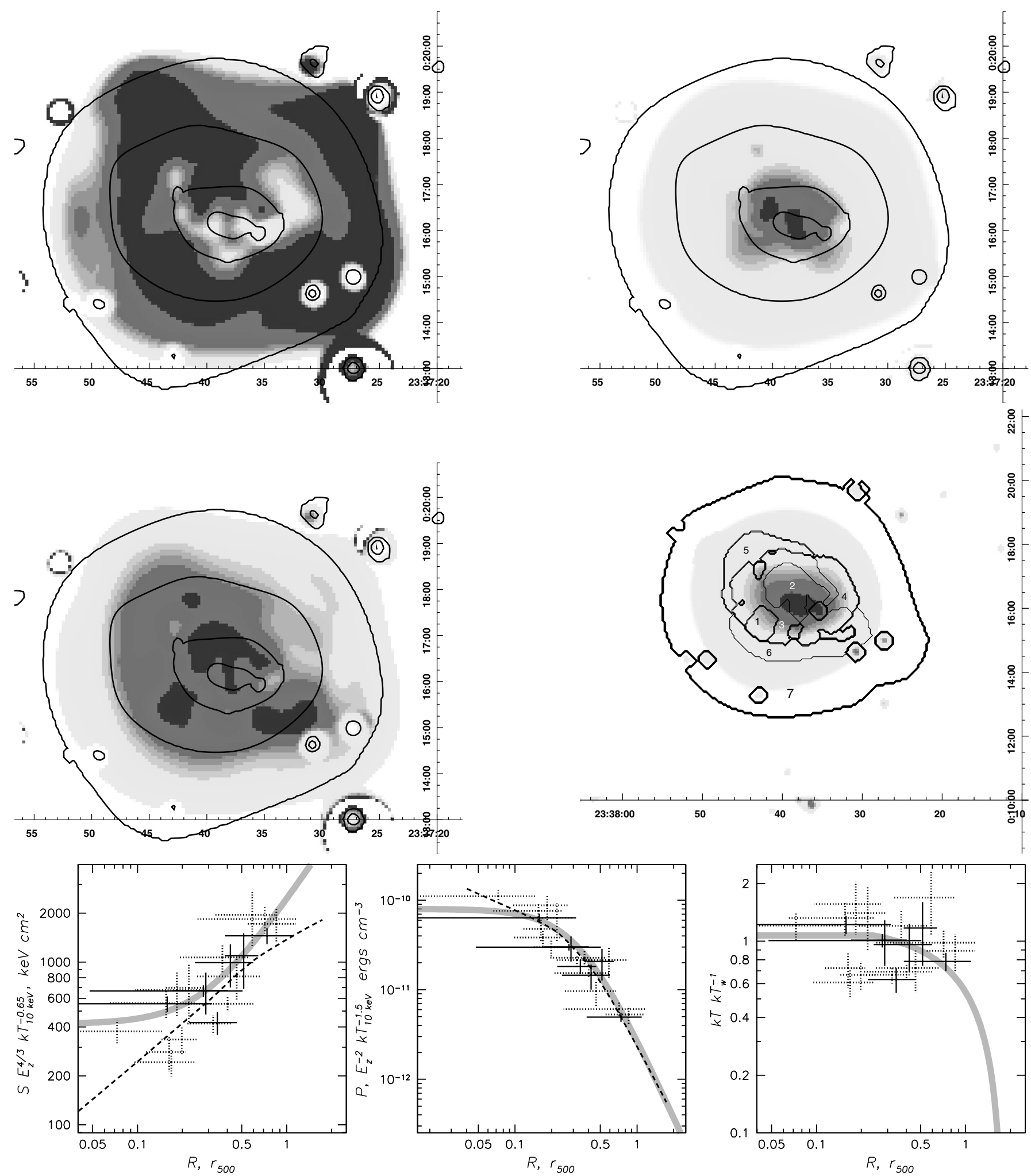

Fig. 12. RXCJ2337.6+0016. Explanations are similar to Fig. 5. The surface brightness image is overlaid with contours showing the spectral extraction regions with numbers corresponding to those in Table 12. 
A. Finoguenov et al.: XMM-Newton maps of DXL clusters, Online Material p 9
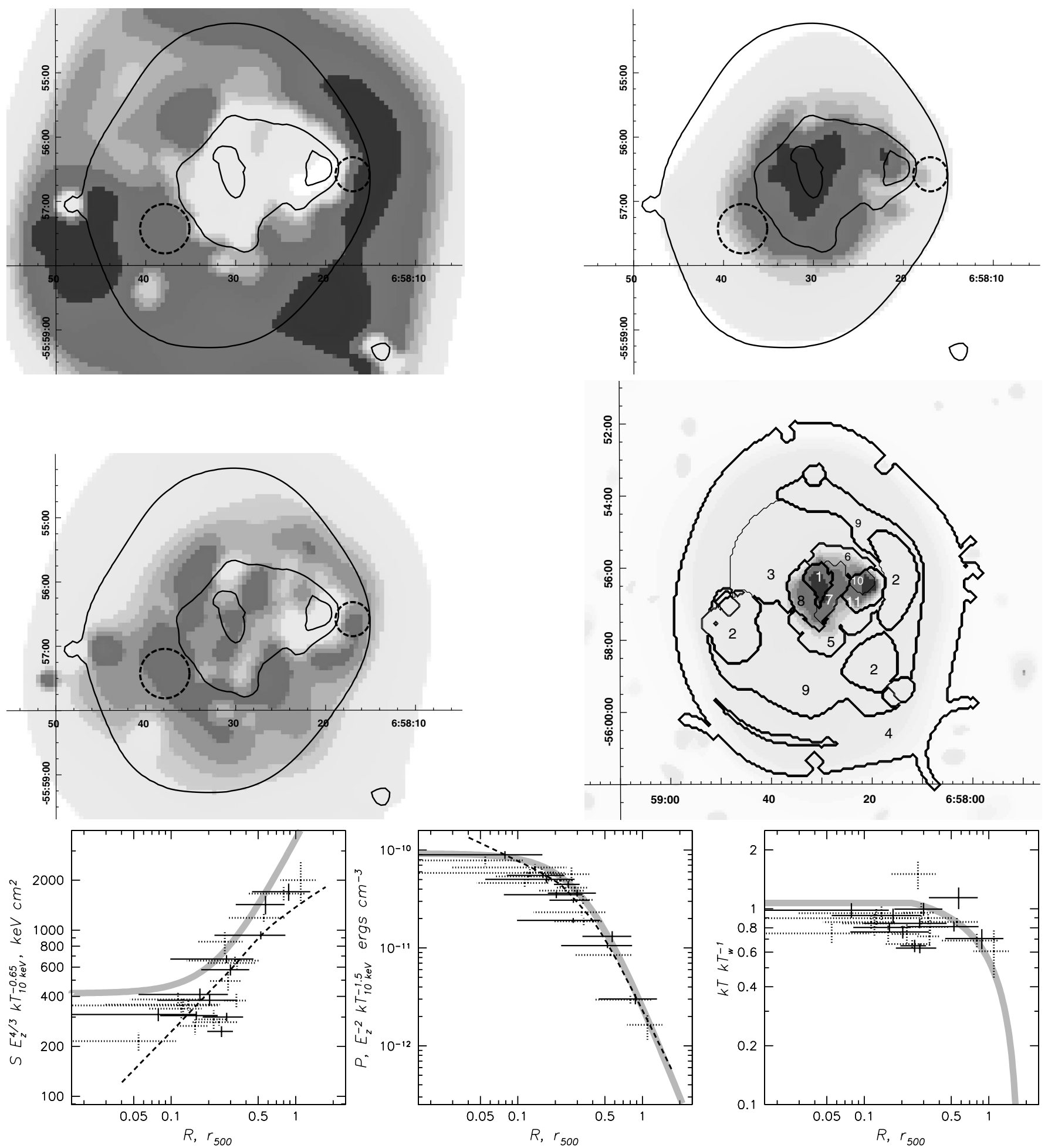

Fig. 13. RXCJ0658-5557. Explanations are similar to Fig. 5. The surface brightness image is overlaid with contours showing the spectral extraction regions with numbers corresponding to those in Table 13. Two dashed line circles indicate the positions of the two peaks revealed in the weak lensing analysis of Clowe et al. (2004). 